\title{
A highly diverse molluscan assemblage associated with eelgrass beds (Zostera marina L.) in the Alboran Sea: Micro-habitat preference, feeding guilds and biogeographical distribution
}

\author{
JOSÉ L. RUEDA, SERGE GOFAS, JAVIER URRA and CARMEN SALAS \\ Departamento de Biología Animal, Universidad de Málaga, Campus de Teatinos s/n, 29071 Málaga, \\ Spain. E-mail: biologiamarina@uma.es
}

\begin{abstract}
SUMMARY: The fauna of molluscs associated with deep subtidal Zostera marina beds (12-16 m) in southern Spain (Alboran Sea) has been characterised in terms of micro-habitat preference, feeding guilds and biogeographical affinity. The species list (162 taxa) is based on sampling completed before the strong eelgrass decline experienced in 2005-2006, using different methods (small Agassiz trawl covering $222 \mathrm{~m}^{2}$ and quadrates covering $0.06 \mathrm{~m}^{2}$ ) and different temporal scales (months, day/night). Dominant epifaunal species are Jujubinus striatus, Rissoa spp. and Smaragdia viridis in the leaf stratum and Nassarius pygmaeus, Bittium reticulatum and Calliostoma planatum on the sediment. Nevertheless, the infauna dominated the epifauna in terms of number of individuals, including mainly bivalves (Tellina distorta, T. fabula, Dosinia lupinus). The epifauna of both the sediment and leaf strata included high numbers of species, probably due to the soft transition between vegetated and unvegetated areas. The dominant feeding guilds were deposit feeders, filter feeders and peryphiton grazers, but ectoparasites (eulimids), seagrass grazers (Smaragdia viridis) and an egg feeder (Mitrella minor) also occurred, unlike in other eelgrass beds of Europe. The molluscan fauna of these Z. marina beds is essentially derived from the local fauna, which includes many widely distributed species along European coasts, with a low representation of strictly Mediterranean or strictly Atlantic species. This fauna is richer than that found in other eelgrass beds of Europe, and deserves important at-
\end{abstract} tention for conservation.

Keywords: bivalves, gastropods, seagrass, biodiversity, Alboran Sea, biogeographical distribution.

RESUMEN: UNA COMUNIDAD ALTAMENTE DIVERSA DE MOLUSCOS ASOCIADOS A PRADERAS DE ZOSTERA MARINA DEL MAR DE ALBORÁN: PREFERENCIAS DE MICRO-HÁBITAT, GRUPOS TRÓFICOS Y DISTRIBUCIÓN BIOGEOGRÁFICA. - La fauna de moluscos asociada a praderas infralitorales profundas de Zostera marina (12-16 m) del sur de España (mar de Alborán) ha sido caracterizada en relación a su micro-hábitat preferente, grupo trófico y distribución biogeográfica. El listado faunístico (162 especies) se obtuvo antes de la fuerte regresión sufrida entre los años 2005 y 2006, usando diferentes métodos (pequeño patín de Agassiz que muestreaba $222 \mathrm{~m}^{2}$ y cuadrantes que muestreaban $0.06 \mathrm{~m}^{2}$ ) y escalas temporales (meses, día/noche). Las especies dominantes asociadas al estrato foliar son Jujubinus striatus, Rissoa spp. y Smaragdia viridis y al sedimento son Nassarius pygmaeus, Calliostoma planatum y Bittium reticulatum, entre otras. No obstante, la endofauna domina a la epifauna, incluyendo principalmente bivalvos como Tellina distorta, T. fabula y Dosinia lupinus. La epifauna asociada al sedimento y al estrato foliar tiene una gran riqueza de especies, probablemente debido a la suave transición entre zonas cubiertas y no cubiertas. Los grupos tróficos dominantes fueron los depositívoros, filtradores o ramoneadores, pero ectoparásitos (eulímidos) y consumidores de fanerógamas marinas (Smaragdia viridis) o de puestas (Mitrella minor) también estuvieron presentes al contrario que en otras praderas de Z. marina de Europa. La fauna de moluscos asociados a Z. marina proviene del gran grupo de especies presentes en la zona, e incluye fundamentalmente especies de amplia distribución a lo largo de las costas europeas, con una baja representación de especies estrictamente Mediterráneas o Atlánticas. Esta fauna es más rica que la encontrada en otras praderas de Z. marina de Europa, lo cual reclama medidas eficientes para una mejor conservación medioambiental de este tipo de hábitat.

Palabras clave: bivalvos, gasterópodos, fanerógamas marinas, biodiversidad, mar de Alborán, distribución biogeográfica. 


\section{INTRODUCTION}

The southern Iberian Peninsula is an area of faunistic confluence due to its location between the Atlantic Ocean, the Mediterranean Sea and northern Africa (Ekman, 1953). The Alboran Sea is located in this area and probably supports the highest marine biodiversity along European coasts (e.g. for Molluscs: van Aartsen et al., 1984; Rueda et al., 2000; Peñas et al., 2006). The molluscs are one of the dominant groups of the marine benthic communities and they contribute largely to the local biodiversity. They are generally strongly associated with different types of habitat such as rocky reefs, soft bottoms and seagrass beds, among others (Hemminga and Duarte, 2000; Luque and Templado, 2004).

The molluscan fauna associated with seagrass beds has received much attention in European seas. Along the Atlantic coasts, the molluscan fauna associated with Zostera marina and Zostera noltii is well known (Jacobs and Huisman, 1982; Jacobs et al., 1983; Currás et al., 1993; Boström and Bonsdorff, 1997, 2000; Frost et al., 1999; Hily and Bouteille, 1999; Fredriksen et al., 2005; Quintas, 2005). On the Mediterranean coasts, the molluscan taxocoenoses have been widely studied for Posidonia oceanica (Ledoyer, 1966; Templado, 1982, 1984a, 1984b; Russo et al., 1984; Gambi et al., 1992; Terlizzi and Russo, 1995; Hergueta, 1996; Scipione et al., 1996), and some studies are also available for Cymodocea nodosa (Scipione et al., 1996; Chemello et al., 1997; Terlizzi and Russo, 1997; Sfriso et al., 2001; Ballesteros et al., 2004). The molluscan taxocoenosis associated with $Z$. marina beds has been less studied (Ledoyer, 1966; Mars, 1966; Çinar et al., 1998; Sfriso et al., 2001; García Raso et al., 2004; Arroyo et al., 2006; Rueda et al., 2008b) in comparison with other areas of its broad biogeographical distribution along cold and temperate areas of the northern Hemisphere, such as the Atlantic coasts of Europe (previously mentioned), North America (Thayer et al., 1975; Marsh, 1973, 1976, Mattila et al., 1999) and Asia (Toyohara et al., 1999; Nakaoka et al., 2001). Eelgrass beds are scarce along the Mediterranean coasts and mostly limited to shallow coastal lagoons (Green and Short, 2003), and this may explain the scarcity of previous studies.

In southern Spain, beds of Z. marina occur in open bays of the Mediterranean coasts (Málaga and Granada) at 6-17 metres depth (Moreno and Guirado, 2003; Rueda et al. 2008a), probably representing the deepest eelgrass beds in Europe. Unfortunately, these beds are experiencing a strong decline as a consequence of, among other factors, illegal trawling (Rueda et al., 2008a). The information on the associated molluscan assemblages is still limited (García Raso et al., 2004; Arroyo et al., 2006; Rueda et al., 2008 b) in comparison with that of $P$. oceanica in the same area (Templado, 1982, 1984a, 1984b; Hergueta, 1996). In three previous papers, we addressed some aspects of the temporal dynamics of the molluscan assemblages in two Aboran Sea eelgrass beds located very close to each other (Cantarriján: Arroyo et al., 2006; Cañuelo: Rueda et al., 2008b; Rueda and Salas, 2008). This study will focus on biological, ecological and distributional aspects of the molluscan fauna found in these eelgrass beds, of which some aspects were briefly outlined in the aforementioned publications. The sampling campaigns were carried out before the strong decline observed in 2005 and 2006 (Rueda et al., 2008a) in those endangered $Z$. marina beds of southern Spain, so this study may be useful as a baseline for a future management of the remaining eelgrass beds and for further studies on the recovery of the fauna to their original levels. Our initial hypothesis is that a very diverse fauna of molluscs inhabits these Z. marina beds located in the Alboran Sea as a result of the biogeographical location, within the context of a faunistic crossroads (between the Atlantic and Mediterranean basins and between Africa and Europe), and a deeper location in relation to the shallower eelgrass beds of other parts of the northern hemisphere.

\section{MATERIAL AND METHODS}

\section{Study area}

All the samples were collected in two different Zostera marina beds within the Marine Protected Area "Paraje Natural de Acantilados de Maro-Cerro Gordo", which is located between the provinces of Málaga and Granada (southern Spain). In 2001, the eelgrass beds had a different degree of coverage and extension at 8-17 metres depth (Bañares-España et $a l ., 2002)$. The studied eelgrass beds were located (1) in front of "Cala de Cantarriján" (Cantarrijan) at 14-16 metres depth $\left(36^{\circ} 44.2^{\prime} \mathrm{N}, 03^{\circ} 46.6^{\prime} \mathrm{W}\right)$, with a patchy distribution (Arroyo et al., 2006) and (2) in front of "Cala de los Cañuelos" (Cañuelo) at 12-14 metres depth $\left(36^{\circ} 44.7^{\prime} \mathrm{N}, 03^{\circ} 47.6^{\prime} \mathrm{W}\right)$, with a higher 


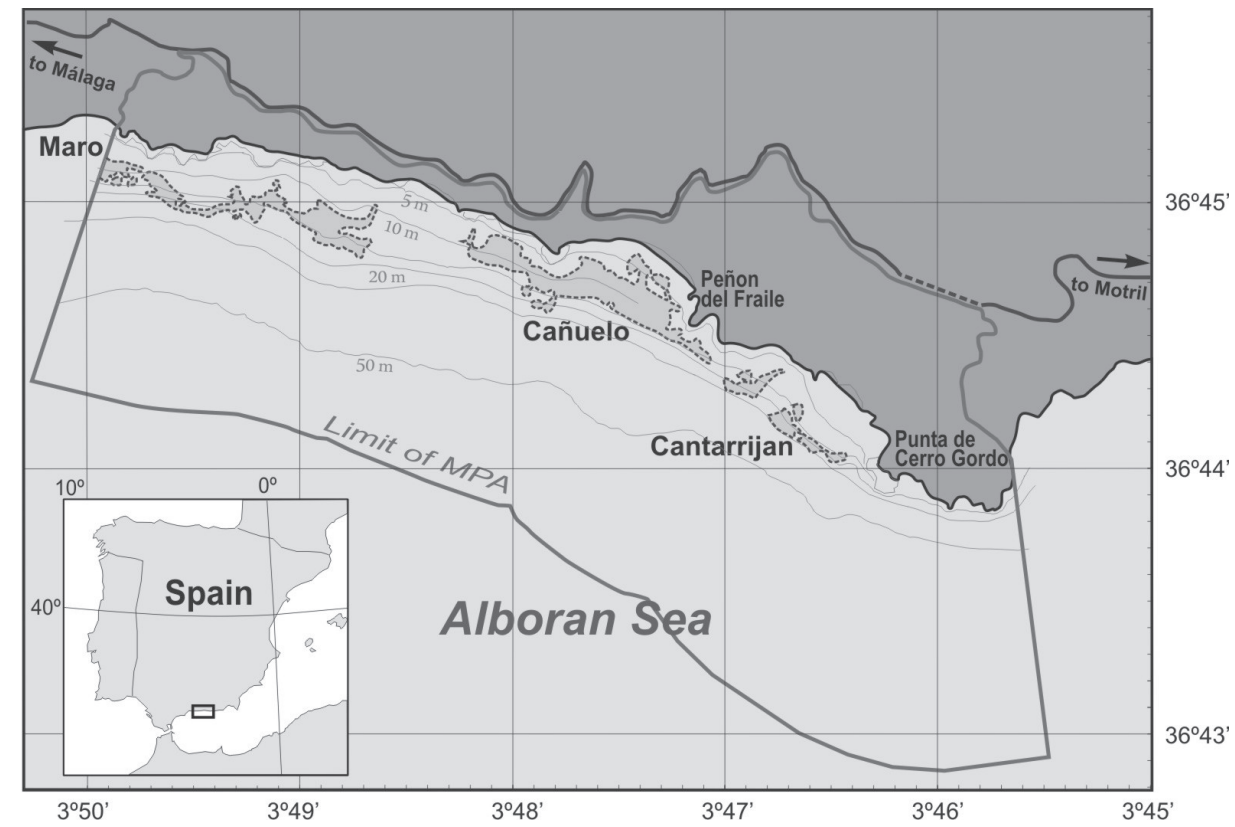

FIG. 1. - Location map of the study area, displaying the presence and distribution of Zostera marina beds within the MPA "Paraje Natural de Acantilados de Maro - Cerro Gordo" based on Bañares-España et al. (2002).

degree of coverage and extension (Rueda et al., 2008b; Rueda and Salas, 2008) (Fig. 1). Information on the characteristics and seasonal dynamics of eelgrass beds in the Alboran Sea has been given in a recent study by Rueda et al. (2008a).

\section{Sampling of epifaunal and infaunal species}

The basis for this study was the set of samples described in Arroyo et al. (2006) and Rueda et al. (2008b), exclusively targeted to the epifauna of the Zostera marina beds, complemented by a further sampling which also encompassed the infauna (Rueda and Salas, 2008). A total of 413574 molluscs belonging to 162 taxa were collected in the different surveys. In those studies, the epifauna and infauna associated with these eelgrass beds was collected using different sampling methods in order to maximise the collection of the different species (abundant or scarce) inhabiting these beds. Samples of epifauna were collected monthly at Cantarrijan (October 1996September 1997; only diurnal samples) and Cañuelo (March 2000-March 2001; diurnal and nocturnal samples) with a small Agassiz trawl with an opening of $72 \mathrm{~cm}$ width and $30 \mathrm{~cm}$ height; the mesh size was $3 \times 3 \mathrm{~mm}$ from knot to knot. On each occasion, the sampling area was around $222 \mathrm{~m}^{2}$, resulting from the width of the Agassiz trawl and the boat speed of 1 knot per hour during 10 minutes. With this method, a total of 40 samples (2-4 monthly replicates) were collected at Cantarrijan and 25 samples (13 diurnal and 12 nocturnal samples) at Cañuelo (Arroyo et al., 2006; Rueda et al., 2008b). Samples of infauna and epifauna were also collected seasonally at Cañuelo (12-14 m depth) in June, September and December 2004 and March 2005 using quadrates $(25$ x $25 \mathrm{~cm}$ : $0.06 \mathrm{~m}^{2}$ per sampled area) and scuba equipment. All Z. marina shoots and $4-5 \mathrm{~cm}$ of the sediment layer were collected inside each quadrate, together with the associated fauna, and placed in cloth bags (mesh size $<0.5 \mathrm{~mm}$ ). For sediment collection, a large plastic spoon ( $15 \mathrm{~cm}$ and $250 \mathrm{ml}$ volume) was used and sediment collected was carefully introduced in the cloth bag that was placed adjacent to one of the sides of the quadrate to minimise loss of sediment material. A total of 6 replicates were collected in September and December and 5 replicates in June and March, covering a sampled seasonal area of between 0.30 (5 rep) and $0.36 \mathrm{~m}^{2}$ (6 rep) (Rueda and Salas, 2008).

In the laboratory, all samples were sorted and molluscan species retained in a $0.5 \mathrm{~mm}$ sieve were identified and counted. The molluscan individuals were labelled and preserved in 70\% ethanol.

\section{Characterisation of the molluscan fauna}

Each species was quantified in each set of samples according to (1) abundance ( $\mathrm{N}$ ind: total number of individuals collected); (2) the frequency index (\%Fr: percentage of samples in which the species is 
TABLE 1. - List and meaning of codes used in the characterisation of the species in relation to their micro-habitat preference, feeding guild and biogeographical distribution.

\begin{tabular}{|c|c|c|}
\hline Category & Code & Comments \\
\hline $\begin{array}{l}\text { Preferential substrate } \\
\text { (Micro-habitat) }\end{array}$ & $\begin{array}{l}\text { DE } \\
\text { EP } \\
\text { HE } \\
\text { HI } \\
\text { SB } \\
\text { SE } \\
\text { SI } \\
\text { VE }\end{array}$ & $\begin{array}{l}\text { Demersal that may bury } \\
\text { Epibionts and ectoparasites } \\
\text { Hard bottoms epifauna } \\
\text { Hard bottoms infauna } \\
\text { Partly buried in soft bottoms } \\
\text { Soft bottoms epifauna } \\
\text { Soft bottoms infauna } \\
\text { Epifauna on vegetated substrates }\end{array}$ \\
\hline Trophic group & $\mathrm{AG}$ & $\begin{array}{l}\text { Herbivores of macroalgae and } \\
\text { epiphytes }\end{array}$ \\
\hline (Feeding guild) & $\begin{array}{l}\text { C } \\
\text { D } \\
\text { E } \\
\text { F } \\
\text { MG } \\
\text { SC } \\
\text { SG } \\
\text { SY } \\
\text { O }\end{array}$ & $\begin{array}{l}\text { Carnivores on mobile preys } \\
\text { Deposit feeders } \\
\text { Ectoparasites and carnivores on } \\
\text { preys without mobility } \\
\text { Filter feeders } \\
\text { Microalgae herbivores } \\
\text { Scavengers } \\
\text { Seagrass-feeding herbivores } \\
\text { Symbiont-bearing species } \\
\text { Egg and spawn feeders }\end{array}$ \\
\hline $\begin{array}{l}\text { Biogeographical } \\
\text { distribution }\end{array}$ & $\begin{array}{l}\mathrm{AF} \\
\mathrm{CN} \\
\mathrm{IM} \\
\mathrm{ME}\end{array}$ & $\begin{array}{l}\text { Western Africa } \\
\text { Canary Islands } \\
\text { Ibero-Moroccan gulf } \\
\text { Mediterranean Sea (those species } \\
\text { which occur only in the Alboran } \\
\text { Sea are excluded from this sector) } \\
\text { Northern Europe } \\
\text { Western Europe }\end{array}$ \\
\hline
\end{tabular}

present) and (3) the dominance index (\%D: percentage of individuals of one particular species from the total). The species were also characterised in terms of their preference for different micro-habitats within the eelgrass bed, their feeding guilds and their biogeographical distribution. For such purpose, several categories within each aspect were established and they are listed in Table 1. Number and percentage of species within each category in relation to the total number of species were calculated.

In relation to the micro-habitat preference, the following categories were considered: (1) hard-bottom infauna (HI), species which bore different types of hard substrata, such as stones or wood in which they live; (2) soft-bottom infauna (SI), mainly bivalves that are permanently buried in the sediment which is colonised by Z. marina; (3) epibionts and ectoparasites (EP), species which may colonise (e.g. epibionts) or feed (e.g. ectoparasites) on generally larger animals; (4) epifauna on vegetated substrates (VE), species which live on Z. marina leaves or macroalgae present within the eelgrass bed (e.g. Asparagopsis armata, Stypocaulon scoparia); (5) hardbottom epifauna (HE), generally found on rocks or shells; (6) organisms partly buried in soft bottoms (SB); (7) demersal species which may bury in the sediment (DE), including species with high mobility such as the cephalopods and (8) soft-bottom epifauna (SE), including gastropod and bivalve species that generally inhabit the sediment layer covered by Z. marina.

According to the feeding guilds, the following categories were considered: (1) carnivores (C), feeding on other mobile organisms, such as molluscs or polychaetes; (2) scavengers (SC), feeding on remains of dead organisms; (3) deposit feeders (D), feeding on organic particles contained in the sediment covered by Z. marina; (4) ectoparasites and specialised carnivores (E), feeding on much larger organisms on which they live during their life cycle; (5) filter feeders $(F)$, capturing the seston particles with their gills and/or with mucous strings; (6) macroalgae grazers (AG); (7) seagrass grazers (SG), ingesting seagrass tissues; (8) microalgal or periphyton grazers (MG), feeding on microalgae (e.g. diatoms) that cover the Z. marina leaves; (9) oophagous feeders (O), including species that feed on egg masses of other organisms; and (10) symbiont-bearing species (SY) for those species in which symbiont bacteria play an important role for obtaining a complementary food source.

Information on the micro-habitat preference and feeding guilds was obtained from the literature available on molluscan fauna associated with seagrass beds (Ledoyer, 1962, 1966; Templado, 1982; Hergueta, 1996; Luque and Templado, 2004; Quintas, 2005) or general literature on mollusc ecology (Fretter and Graham, 1962; Tebble, 1966; Morton, 1967; Graham, 1971; Bouchet et al., 1979; Nordsieck and García-Talavera, 1979; Kohn, 1983; Russell-Hunter, 1983; Luque, 1984, 1986; Templado et al., 1993; Hayward and Ryland, 1995; Gómez-Rodríguez and Pérez-Sánchez, 1997; Beesley et al., 1998; Rueda, 2007). Moreover, information on preference of species for different micro-habitats within the eelgrass bed and feeding was obtained during collection of samples and laboratory observations.

The characterisation of the species according to their biogeographical distribution is problematic, due to the lack of consensus on geographical areas that have been established by different authors, in most cases according to oceanographic characteristics (Ekman, 1953; Briggs, 1974; Longhurst, 1998). In order to handle the geographical range with more detail, geographical sectors were established 
on a finer scale as follows: (0) Alboran Sea, all the species found in this study; (1) Mediterranean Sea (ME), excluding from this category those species that do not generally occur east of the Alboran Sea; (2) Ibero-Moroccan Gulf (IM), including the southern coasts of Portugal, Atlantic coasts of Andalusia (southwestern Spain) and Morocco according to the faunistic lists given by Pallary (1920), Rueda et al. (2000), Rueda et al. (2001), Rueda and Salas (2003 a, b) and to unpublished data of the Algarve 1988 expedition of Muséum National d'Histoire Naturelle, Paris; (3) western Europe (WE), from Portugal to the southern coasts of United Kingdom, according to the species listed in Nobre (1940), Tebble (1966), Graham (1971), Bouchet et al. (1979), Thompson and Brown (1976), Rolán (1983) and Rolán et al. (1990); (4) northern Europe (NE), from the southern coasts of the United Kingdom to Scandinavia, according to the species listed in Høisæter (1985) and Hansson (1998); (5) the Canary Islands (CN), according to the works by Nordsieck and García-Talavera (1979) and Gómez-Rodríguez and Pérez-Sánchez (1997); and (6) western Africa (AF), from Mauritania to tropical western African coasts, according to the information given by Gofas and Zenetos (2003) on western African species that also occur in the Alboran Sea. The presence of the different species in each geographical sector was annotated and their chorotypes were established. For this purpose, a cluster was performed using the Bray-Curtis similarity index (Bray and Curtis, 1957), in which species with a similar biogeographical range were grouped. In this analysis, qualitative data (presence/absence of species in each sector) was used without any further transformation and standardisation. This multivariate analysis was carried out using the PRIMER from Plymouth Marine Laboratory, UK (Clarke and Warwick, 1994).

\section{RESULTS}

\section{Composition and structure of molluscan fauna}

The gastropods were the most diversified group with 99 species, followed by bivalves (57 spp.), cephalopods (4 spp.) and polyplacophorans (2 spp.). The best represented families were Conidae (10 spp.), Rissoidae (7 spp.), Pyramidellidae (7 spp.), Eulimidae (5 spp.), Trochidae (5 spp.) and Nassariidae (5 spp.) for gastropods and Veneridae (9 spp.),
Mytilidae (6 spp.), Cardidae (6 spp.) and Tellinidae (6 spp.) for bivalves. The list of the 162 taxa is presented in Table 2, and some of the most characteristic species are shown in Figures 2 to 7.

There was an extensive, but not total, overlap of the species composition between the three surveys. Eighty taxa were collected in the Zostera marina bed of Cantarrijan using the small Agassiz trawl, whereas 116 were collected in the dense bed located in Cañuelo with the same sampling methodology and 85 using the quadrates. A total of 39 species were collected in all different surveys such as the gastropods Calliostoma planatum, Jujubinus striatus, Smaragdia viridis, Bittium latreili, B. reticulatum, Rissoa membranacea, $R$. monodonta, Nassarius cuvierii, $N$. incrassatus, $N$. pygmaeus, $N$. reticulatus and Mitrella minor and the bivalves Modiolarca subpicta, Musculus costulatus, Parvicardium scriptum, Spisula subtruncata, Chamelea gallina, Venerupis aurea and Pitar rudis, among other species. These species generally displayed high values of the frequency $(\% \mathrm{~F})$ and/or dominance index $(\% \mathrm{D})$ (Table $2)$. In fact, the gastropods $J$. striatus, $B$. reticulatum, $R$. membranacea, $R$. monodonta, S. viridis, $N$. pygmaeus and $M$. minor were the dominant epifaunal species in all samples.

With a different sampling methodology such as the quadrates, a total of 28 species were added to the faunistic list obtained in previous samplings. This group includes some small gastropods such as Eulima glabra, Cylichnina crossei, Pyrunculus hoernesii and Retusa minutissima and mostly bivalves such as Solemya togata, Scacchia oblonga, Sportella recondita, Tellina spp., Gari spp., Dosinia lupinus and Thracia villosiuscula. Some of these species displayed either high values of $\% \mathrm{D}$ or $\% \mathrm{~F}$, such as Tellina distorta, D. lupinus and T. fabula, indicating that they are common and dominant in the eelgrass bed, or only high values of $\% \mathrm{~F}$, such as $C$. crossei and $S$. togata, indicating that they occur permanently in the bed throughout the year but at low densities. No cephalopods and large-sized gastropods (e.g. Bolma rugosa, Aplysia spp.) were collected using this sampling technique (Table 2).

\section{Micro-habitat and feeding guilds}

The composition of the malacofauna is dominated, regarding the preference of species for different types of micro-habitats (Tables 2 and 3), by the following categories: 
TABLE 2. - Faunistic list of molluscs found in Zostera marina beds in southern Spain, with quantitative data from Arroyo et al. (2006) (Cantarriján, Agassiz), Rueda et al. (2008b) (Cañuelo, Agassiz) and Rueda and Salas (2008) (Cañuelo, quadrates). Codes for preferred micro-habitat within the eelgrass bed (MH), feeding guild (FG) and biogeographical distribution as listed in Table 1 . Total number of individuals collected ( $\mathrm{N}$ ind) and values of frequency index (among brackets) are also indicated for each species in each of the surveys. Dominance index for each species can be calculated as percentage of $\mathrm{N}$ ind in relation to total number of molluscs collected in each survey. Species marked with $*$ are illustrated in Figures 2 to 7 . UN: Unknown.

\begin{tabular}{|c|c|c|c|c|c|c|c|c|c|c|c|}
\hline \multirow[t]{2}{*}{ Species } & \multirow[t]{2}{*}{ MH } & \multirow[t]{2}{*}{ FG } & \multicolumn{6}{|c|}{ Biogeographical distribution } & \multirow{2}{*}{$\begin{array}{c}\text { Cantarrijan } \\
\text { Agassiz }\end{array}$} & \multirow{2}{*}{$\begin{array}{l}\text { Cañuelo } \\
\text { Agassiz }\end{array}$} & \multirow[b]{2}{*}{ Quadrates } \\
\hline & & & $\mathrm{ME}$ & IM & WE & $\mathrm{NE}$ & $\mathrm{CN}$ & $\mathrm{AF}$ & & & \\
\hline \multicolumn{12}{|l|}{ POLYPLACOPHORANS } \\
\hline Lepidochitona cinerea (Linnaeus, 1767) & $\mathrm{HE}$ & MG & + & + & + & + & & & & & $1(4.5)$ \\
\hline Chiton olivaceus (Spengler, 1797) & $\mathrm{HE}$ & MG & + & + & & & & & $1(2.5)$ & & \\
\hline \multicolumn{12}{|l|}{ GASTROPODS } \\
\hline Calliostoma planatum Pallary, $1900 *$ & $\mathrm{SE}$ & $\mathrm{E}$ & & & & & & & $236(75)$ & $8189(100)$ & $5(18.2)$ \\
\hline Calliostoma zizyphinum (Linnaeus, 1758) & $\mathrm{SE}$ & $\mathrm{E}$ & + & + & + & + & & & & $10(28)$ & \\
\hline Gibbula magus (Linnaeus, 1758) & $\mathrm{SE}$ & MG & + & + & + & & + & & $19(20)$ & & \\
\hline Gibbula racketti (Payraudeau, 1826) & $\mathrm{HE}$ & MG & + & & & & & & & $2(8)$ & \\
\hline Gibbula varia (Linnaeus, 1758) & $\mathrm{HE}$ & MG & + & & & & & & & 1(4) & \\
\hline Jujubinus striatus (Linnaeus, 1758) * & VE & MG & + & + & + & + & & & $32166(100)$ & $241712(100)$ & $123(95.5)$ \\
\hline Phorcus richardi (Payraudeau, 1826) & $\mathrm{HE}$ & MG & + & & & & & & $2(5)$ & & \\
\hline Tricolia pullus (Linnaeus, 1758) & VE & MG & + & + & + & + & + & & & $75(80)$ & $1(4.5)$ \\
\hline Tricolia tenuis (Michaud, 1829) & $\mathrm{VE}$ & MG & + & + & & & & & & $29(52)$ & \\
\hline Bolma rugosa (Linnaeus, 1767) & $\mathrm{SE}$ & MG & + & + & & & + & & $16(30)$ & $26(48)$ & \\
\hline Smaragdia viridis (Linnaeus, 1758) * & $\mathrm{VE}$ & SG & + & & & & + & & $862(87.5)$ & $1795(100)$ & $21(45.5)$ \\
\hline Bittium latreillii (Payraudeau, 1826) * & SE & MG & + & + & + & & + & & $32(30)$ & $368(100)$ & $5(18.2)$ \\
\hline Bittium reticulatum (da Costa, 1778) * & $\mathrm{SE}$ & MG & + & + & + & + & & & $571(67.5)$ & $3227(100)$ & $72(95.5)$ \\
\hline \multicolumn{12}{|l|}{ Bittium submamillatum (de Rayneval and } \\
\hline Ponzi, 1854) & $\mathrm{SE}$ & MG & + & + & & & & & $1(2.5)$ & & $1(4.5)$ \\
\hline Turritella communis Risso, 1826 & $\mathrm{SE}$ & $\mathrm{F}$ & + & + & + & + & & & $1(2.5)$ & & \\
\hline Turritella turbona Monterosato, 1877 & $\mathrm{SE}$ & $\mathrm{F}$ & + & + & & & & & $3(7.5)$ & $3(8)$ & \\
\hline Triphoridae & $\mathrm{EP}$ & $\mathrm{E}$ & & & & & & & $1(2.5)$ & $89(48)$ & \\
\hline Cerithiopsis jeffreysi Watson, 1874 & $\mathrm{EP}$ & $\mathrm{E}$ & + & + & & & + & & & 2(4) & \\
\hline Cerithiopsis tubercularis (Montagu, 1803) & $\mathrm{EP}$ & $\mathrm{E}$ & + & + & + & + & & & & $6(16)$ & \\
\hline Cirsotrema cochlea (Sowerby G. B. II, 1844) & $\mathrm{EP}$ & $\mathrm{E}$ & & + & & & & + & & $1(4)$ & $1(4.5)$ \\
\hline Epitonium commune (Lamarck, 1822) & $\mathrm{EP}$ & $\mathrm{E}$ & + & + & + & + & & & & $2(8)$ & $6(22.7)$ \\
\hline Epitonium turtonis (Turton, 1819) & $\mathrm{EP}$ & $\mathrm{E}$ & + & + & + & + & & & $4(7.5)$ & & $1(4.5)$ \\
\hline Crinophtheiros sp. & $\mathrm{EP}$ & $\mathrm{E}$ & & & & & & & & $3(12)$ & $1(4.5)$ \\
\hline Eulima glabra (da Costa, 1778) & $\mathrm{EP}$ & $\mathrm{E}$ & + & + & + & + & & & & & $1(4.5)$ \\
\hline Melanella sp. 1 & $\mathrm{EP}$ & $\mathrm{E}$ & & & & & & & & $2(4)$ & \\
\hline Melanella sp. 2 & $\mathrm{EP}$ & $\mathrm{E}$ & & & & & & & & $1(4)$ & \\
\hline Vitreolina philippi (de Rayneval and & & & & & & & & & & & \\
\hline Ponzi, 1854) & $\mathrm{EP}$ & E & + & + & + & + & + & & & $8(20)$ & $2(9.1)$ \\
\hline Skeneopsis planorbis (Fabricius, 1780) & VE & MG & + & + & + & + & & & & $3(12)$ & $2(9.1)$ \\
\hline Pusillina inconspicua (Alder, 1844) * & $\mathrm{VE}$ & MG & + & + & + & + & & & $23(7.5)$ & $1133(84)$ & $11(36.4)$ \\
\hline Pusillina philippi (Aradas and Maggiore, 1844) * & $\mathrm{VE}$ & MG & + & & & & & & $4(2.5)$ & $52(24)$ & \\
\hline Pusillina cf. radiata (Philippi, 1836) & $\mathrm{VE}$ & MG & + & + & & & & & $4(10)$ & & \\
\hline Rissoa guerinii Récluz, 1843 * & $\mathrm{VE}$ & MG & + & + & + & & & & & $22(44)$ & \\
\hline Rissoa membranacea (Adams J., 1800) * & $\mathrm{VE}$ & MG & & + & + & + & & & $4418(95)$ & $6425(100)$ & $11(27.3)$ \\
\hline Rissoa monodonta Philippi, $1836 *$ & $\mathrm{VE}$ & MG & + & & & & & & $637(80)$ & $1551(100)$ & $6(27.3)$ \\
\hline Rissoa violacea Desmarest, $1814 *$ & $\mathrm{VE}$ & MG & + & & & & & & & $87(80)$ & \\
\hline Barleeia unifasciata (Montagu, 1803) & $\mathrm{VE}$ & $\mathrm{AG}$ & + & + & + & + & + & & & $4(12)$ & \\
\hline Aporrhais pespelecani (Linnaeus, 1758) & $\mathrm{SE}$ & $\mathrm{F}$ & + & + & + & + & & & $41(25)$ & $1(4)$ & $1(4.5)$ \\
\hline Calyptraea chinensis (Linnaeus, 1758) & SE & $\mathrm{F}$ & + & + & + & + & & & $102(60)$ & $52(72)$ & $19(45.5)$ \\
\hline Crepidula unguiformis Lamarck, 1822 & $\mathrm{SE}$ & $\mathrm{F}$ & + & & & & & & & $2(8)$ & \\
\hline Euspira macilenta (Philippi, 1844) & SB & $\mathrm{C}$ & + & & & & & & $2(2.5)$ & $1(4)$ & \\
\hline Euspira pulchella (Risso, 1826) & SB & $\mathrm{C}$ & + & + & + & + & & & $13(32.5)$ & $11(36)$ & $6(27.3)$ \\
\hline Payraudeautia intricata (Donovan, 1804) & SB & $\mathrm{C}$ & + & + & & & & & $2(5)$ & & \\
\hline Tectonatica filosa (Philippi, 1845) & SB & $\mathrm{C}$ & + & + & & & & + & $8(15)$ & $2(8)$ & $2(9.1)$ \\
\hline Phalium undulatum (Gmelin, 1791) & SB & $\mathrm{C}$ & + & + & & & + & & & $1(4)$ & \\
\hline Hexaplex trunculus (Linnaeus, 1758) & $\mathrm{SE}$ & $\mathrm{C}$ & + & + & & & + & & $3(7.5)$ & 1(4) & \\
\hline Ocenebra erinaceus (Linnaeus, 1758) & $\mathrm{SE}$ & $\mathrm{C}$ & + & + & + & + & & & $6(12.5)$ & $12(32)$ & \\
\hline Ocinebrina aciculata (Lamarck, 1822) & $\mathrm{SE}$ & $\mathrm{C}$ & + & + & + & + & & & $10(20)$ & $21(52)$ & \\
\hline Orania fusulus (Brocchi, 1814) & SE & $\mathrm{C}$ & + & + & & & + & + & $2(5)$ & & \\
\hline Gibberula miliaria (Linnaeus, 1758) & $\mathrm{HE}$ & $\mathrm{C}$ & + & + & & & & & $3(7.5)$ & & \\
\hline Cymbium olla (Linnaeus, 1758) & $\mathrm{SE}$ & $\mathrm{C}$ & & + & & & & & & $1(4)$ & \\
\hline Pollia dorbignyi (Payraudeau, 1826) & $\mathrm{HE}$ & $\mathrm{C}$ & + & & & & & & & $9(24)$ & \\
\hline Nassarius cuvierii (Payraudeau, 1826) * & $\mathrm{SE}$ & $\mathrm{SC}$ & + & + & & & & & $2(5)$ & $16(36)$ & $7(18.2)$ \\
\hline Nassarius heynemanni (Maltzan, 1884) & SE & $\mathrm{SC}$ & & + & & & & + & $1(2.5)$ & & \\
\hline Nassarius incrassatus (Ström, 1768) * & $\mathrm{SE}$ & $\mathrm{SC}$ & + & + & + & + & + & & $104(47.5)$ & 803(100) & $6(22.7)$ \\
\hline Nassarius pygmaeus (Lamarck, 1822) * & $\mathrm{SE}$ & $\mathrm{SC}$ & + & + & + & + & & & $2591(87.5)$ & $68456(100)$ & $114(95.5)$ \\
\hline Nassarius reticulatus (Linnaeus, 1758) * & $\mathrm{SE}$ & $\mathrm{SC}$ & + & + & + & & & & $274(62.5)$ & $1377(84)$ & $50(95.5)$ \\
\hline Mitrella minor (Scacchi, 1836) * & $\mathrm{VE}$ & $\mathrm{O}$ & + & + & & & & & $1799(82.5)$ & 28011(100) & $27(77.3)$ \\
\hline Fusinus pulchellus (Philippi, 1844) & $\mathrm{SE}$ & $\mathrm{C}$ & + & + & & & & & $6(12.5)$ & $16(48)$ & \\
\hline Cancellaria cancellata (Linnaeus, 1767) & SE & $\mathrm{C}$ & & + & & & & + & $82(45)$ & $5(16)$ & \\
\hline
\end{tabular}


TABLE 2 (cont.). - Faunistic list of molluscs found in Zostera marina beds in southern Spain, with quantitative data from Arroyo et al. (2006) (Cantarriján, Agassiz), Rueda et al. (2008b) (Cañuelo, Agassiz) and Rueda and Salas (2008) (Cañuelo, quadrates).

\begin{tabular}{|c|c|c|c|c|c|c|c|c|c|c|c|}
\hline \multirow[t]{2}{*}{ Species } & \multirow[t]{2}{*}{ MH } & \multirow[t]{2}{*}{ FG } & \multicolumn{6}{|c|}{ Biogeographical distribution } & \multirow{2}{*}{$\begin{array}{c}\text { Cantarrijan } \\
\text { Agassiz }\end{array}$} & \multirow{2}{*}{$\begin{array}{l}\text { Cañuelo } \\
\text { Agassiz }\end{array}$} & \multirow[b]{2}{*}{ Quadrates } \\
\hline & & & ME & IM & WE & NE & $\mathrm{CN}$ & $\mathrm{AF}$ & & & \\
\hline Bela costulata (Risso, 1826) * & SE & $\mathrm{C}$ & + & + & + & + & & & $25(25)$ & $7(24)$ & $4(13.6)$ \\
\hline Bela laevigata (Philippi, 1836) * & SE & $\mathrm{C}$ & + & + & & & & & $8(7.5)$ & $3(12)$ & $2(9.1)$ \\
\hline Bela nebula (Montagu, 1803) * & SE & $\mathrm{C}$ & & + & + & + & & & $18(20)$ & $6(20)$ & $4(18.2)$ \\
\hline Bela sp. $1 *$ & SE & $\mathrm{C}$ & & & & & & & $28(35)$ & $16(44)$ & $9(27.3)$ \\
\hline Comarmondia gracilis (Montagu, 1803) & SE & $\mathrm{C}$ & + & + & + & + & + & & & $5(12)$ & \\
\hline Mangelia attenuata (Montagu, 1803) * & SE & $\mathrm{C}$ & + & + & + & + & & & $20(35)$ & $3(8)$ & $3(13.6)$ \\
\hline Mangelia paciniana (Calcara, 1839) * & SE & $\mathrm{C}$ & + & & & & & & $2(5)$ & $2(8)$ & \\
\hline Mangelia unifasciata Deshayes, $1835 *$ & SE & $\mathrm{C}$ & + & + & & & & & $14(25)$ & $26(48)$ & $3(13.6)$ \\
\hline Raphitoma cordieri (Payraudeau, 1826) & $\mathrm{HE}$ & $\mathrm{C}$ & + & & & & & & & $1(4)$ & \\
\hline Raphitoma linearis (Montagu, 1803) & $\mathrm{HE}$ & $\mathrm{C}$ & + & + & + & + & & & & 1(4) & \\
\hline Crassopleura maravignae (Bivona, 1838) & SE & $\mathrm{C}$ & + & & & & + & & $3(7.5)$ & & \\
\hline Haedropleura septangularis (Montagu, 1803) & 3) $\mathrm{SE}$ & $\mathrm{C}$ & + & + & + & + & & & $1(2.5)$ & $5(16)$ & \\
\hline Rissoella diaphana (Alder, 1848) & VE & MG & + & + & + & + & & & & $1(4)$ & \\
\hline Ammonicera rota (Forbes and Hanley, 1853) & $\mathrm{VE}$ & MG & + & + & + & + & & & & $1(4)$ & \\
\hline Chrysallida brusinai (Cossmann, 1921) & $\mathrm{EP}$ & $\mathrm{E}$ & + & + & + & & & & & $5(16)$ & \\
\hline Chrysallida emaciata (Brusina, 1866) & $\mathrm{EP}$ & $\mathrm{E}$ & + & & & & & & & $8(20)$ & \\
\hline Odostomia conspicua Alder, 1850 & $\mathrm{EP}$ & $\mathrm{E}$ & & + & + & + & & & & $1(4)$ & \\
\hline Odostomia turrita Hanley, $1844 *$ & $\mathrm{EP}$ & $\mathrm{E}$ & + & + & + & + & & & & $372(84)$ & \\
\hline Odostomia unidentata (Montagu, 1803) & EP & $\mathrm{E}$ & + & + & + & + & & & & $25(40)$ & \\
\hline Turbonilla rufa (Philippi, 1836) & EP & $\mathrm{E}$ & + & + & + & + & & & & & $1(4.5)$ \\
\hline Turbonilla sp. & EP & $\mathrm{E}$ & & & & & & & & $2(4)$ & $1(4.5)$ \\
\hline Acteon tornatilis (Linnaeus, 1758) & SB & $\mathrm{C}$ & + & + & + & + & & & $3(5)$ & $1(4)$ & \\
\hline Cylichnina crossei (Bucquoy, Dautzenberg & & & & & & & & & & & \\
\hline and Dollfus, 1886) * & SB & $\mathrm{C}$ & + & & & & & & & & $35(68.2)$ \\
\hline Pyrunculus hoernesii (Weinkauff, 1866) * & SB & $\mathrm{C}$ & + & + & & & & & & & 3(13.6) \\
\hline Retusa minutissima (Monterosato, 1878) * & SB & $\mathrm{C}$ & + & & & & & & & & $1(4.5)$ \\
\hline Volvulella acuminata (Bruguière, 1792) & SB & $\mathrm{C}$ & + & + & & & & & & & $2(9.1)$ \\
\hline Ringicula auriculata (M. de la Groye, 1811) & SB & $\mathrm{C}$ & + & + & & & & & & & $2(9.1)$ \\
\hline Bulla striata Bruguière, $1792 *$ & SB & $\mathrm{C}$ & + & + & & & + & + & & $14(8)$ & \\
\hline Haminoea hydatis (Linnaeus, 1758) & SE & AG & + & + & + & & & & $4(7.5)$ & $29(24)$ & $2(9.1)$ \\
\hline Philine aperta (Linnaeus, 1758) & SB & $\mathrm{C}$ & + & + & + & + & & & & $2(8)$ & $2(9.1)$ \\
\hline Aglaja tricolorata Renier, 1807 & SB & $\mathrm{C}$ & + & + & & & + & & $1(2.5)$ & & \\
\hline Berthella cf aurantiaca (Risso, 1818) & SE & $\mathrm{E}$ & + & + & + & & & & & 2(4) & \\
\hline Pleurobranchaea meckelii Leue, 1813 & SE & $\mathrm{C}$ & + & + & & & & + & $1(2.5)$ & $35(36)$ & \\
\hline Aplysia parvula Guilding in Mørch, 1863 & VE & AG & & & & & & & $1(2.5)$ & $22(52)$ & \\
\hline Aplysia punctata (Cuvier, 1803) & SE & AG & + & + & + & & & & $7(2.5)$ & $185(56)$ & \\
\hline Trapania cf. lineata Haefelfinger, 1960 & $\mathrm{HE}$ & $\mathrm{E}$ & + & + & & & & & $3(2.5)$ & & \\
\hline Polycera quadrilineata (O. F. Müller, 1776) & VE & $\mathrm{E}$ & + & + & + & + & & + & $1(2.5)$ & $138(20)$ & \\
\hline Hancockia uncinata (Hesse, 1872) & $\mathrm{VE}$ & $\mathrm{E}$ & + & + & + & & & & & $10(28)$ & $1(4.5)$ \\
\hline Doto sp. & VE & $\mathrm{E}$ & & & & & & & $6(2.5)$ & $22(8)$ & \\
\hline Williamia gussonii (Costa O. G., 1829) & $\mathrm{HE}$ & AG & + & + & & & + & & & $17(44)$ & $1(4.5)$ \\
\hline BIVALVES & & & & & & & & & & & \\
\hline Nucula nitidosa Winckworth, 1930 & SI & $\mathrm{D}$ & + & + & + & + & & & & & $5(22.7)$ \\
\hline Nuculana pella (Linnaeus, 1767) & SI & $\mathrm{D}$ & + & + & & & & + & & & $3(13.6)$ \\
\hline Solemya togata (Poli, 1791)* & SI & SY & + & & & & + & & & & $23(81.8)$ \\
\hline Glycymeris violacescens (Lamarck, 1819) & SI & $\mathrm{F}$ & + & + & & & & & & & $8(31.8)$ \\
\hline Gregariella semigranata (Reeve, 1858) * & SE & $\mathrm{F}$ & & + & + & & + & & & $16(28)$ & \\
\hline Modiolarca subpicta (Cantraine, 1835) * & SE & $\mathrm{F}$ & + & + & + & + & + & & 137(30) & $327(92)$ & $2(9.1)$ \\
\hline Modiolus adriaticus (Lamarck, 1819) & SE & $\mathrm{F}$ & + & + & + & & + & & & $14(24)$ & $4(13.6)$ \\
\hline Musculus costulatus (Risso, 1826) * & SE & $\mathrm{F}$ & + & + & + & + & + & & $9(7.5)$ & $123(52)$ & $4(18.2)$ \\
\hline Mytilaster minimus (Poli, 1795) * & SE & $\mathrm{F}$ & + & + & + & & + & & $72(10)$ & 105(68) & $8(27.3)$ \\
\hline Mytilus galloprovincialis Lamarck, 1819 & SE & $\mathrm{F}$ & + & + & + & & & & & $10(4)$ & \\
\hline Aequipecten opercularis (Linnaeus, 1758) * & SE & $\mathrm{F}$ & + & + & + & + & + & & & $37(20)$ & 1(4.5) \\
\hline Chlamys flexuosa $($ Poli,1795) * & SE & $\mathrm{F}$ & + & + & & & + & & $1(2.5)$ & $67(40)$ & $10(31.8)$ \\
\hline Chlamys varia (Linnaeus, 1758) & SE & $\mathrm{F}$ & + & + & + & + & + & & & $5(16)$ & \\
\hline Pecten maximus (Linnaeus, 1758) & SE & $\mathrm{F}$ & + & + & + & + & + & & $1(2.5)$ & 2(4) & \\
\hline Anomia ephippium Linnaeus, 1758 * & EP & $\mathrm{F}$ & + & + & + & + & + & & $239(60)$ & $583(100)$ & 112(40.9) \\
\hline Limea loscombii (Sowerby G. B. I, 1824) & SE & $\mathrm{F}$ & + & + & + & + & + & & & 4(12) & \\
\hline Lucinella divaricata (Linnaeus, 1758) * & SI & SY & + & + & + & & + & & & & $114(81.8)$ \\
\hline Scacchia oblonga (Philippi, 1836) * & SI & UN & + & + & & & & & & & 19(40.9) \\
\hline Kellia suborbicularis (Montagu, 1803) & SI & $\mathrm{F}$ & + & + & + & + & + & & & $3(8)$ & \\
\hline Kurtiella bidentata (Montagu, 1803) * & SI & $\mathrm{F}$ & + & + & + & + & & & & $11(20)$ & $46(68.2)$ \\
\hline Tellimya ferruginosa (Montagu, 1808) & SI & $\mathrm{F}$ & + & + & + & + & + & & & & $1(4.5)$ \\
\hline Sportella recondita (Fischer P., 1872) & SI & UN & + & + & + & & & & & & $2(9.1)$ \\
\hline Digitaria digitaria (Linnaeus, 1758) & SI & $\mathrm{F}$ & + & + & & & & & $2(5)$ & & \\
\hline Acanthocardia aculeata (Linnaeus, 1758) & SI & $\mathrm{F}$ & + & + & + & + & + & & $1(2.5)$ & & \\
\hline Acanthocardia tuberculata (Linnaeus, 1758) & SI & $\mathrm{F}$ & + & + & + & & + & & & 1(4) & $1(4.5)$ \\
\hline Papillicardium papillosum (Poli, 1791) * & SI & $\mathrm{F}$ & + & + & + & & + & & $1(2.5)$ & $2(8)$ & $3(13.6)$ \\
\hline Parvicardium exiguum (Gmelin, 1791) & SI & $\mathrm{F}$ & + & + & + & + & + & & & $4(12)$ & \\
\hline
\end{tabular}


TABLE 2 (cont.). - Faunistic list of molluscs found in Zostera marina beds in southern Spain, with quantitative data from Arroyo et al. (2006) (Cantarriján, Agassiz), Rueda et al. (2008b) (Cañuelo, Agassiz) and Rueda and Salas (2008) (Cañuelo, quadrates).

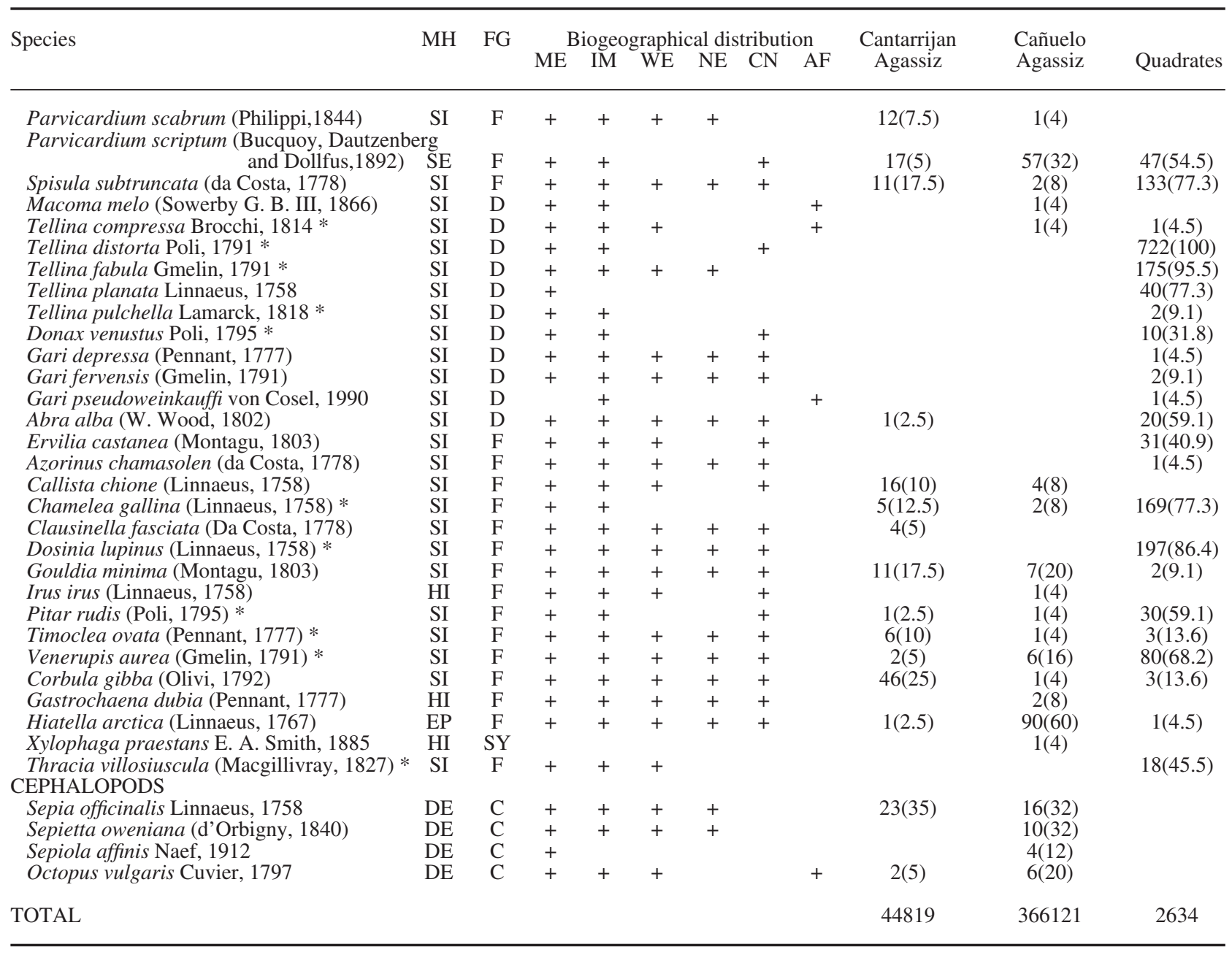

(1) epifaunal species on soft bottoms (SE, Fig. 3), mostly including gastropods of families Conidae (11 spp.) and Nassariidae (4 spp.) and some bivalves of families Mytilidae (6 spp) and Pectinidae (4 spp.). Some of the species that are generally linked to the sediment stratum, such as Calliostoma planatum (Fig. 2), Nassarius spp. and Bittium spp. may also display vertical movement towards the leaf stratum for feeding or spawning during their life cycle;

(2) infaunal species (SI, Figs. 6 and 7), mostly bivalves, such as venerids (9 spp.), tellinids (6 spp.) and cardids ( 6 spp.) and species that are common in the anoxic stratum of the sediment such as Solemya togata or Lucinella divaricata;

(3) epifaunal species associated with eelgrass or macroalgae (VE, Fig. 2), which are mostly gastropods such as Jujubinus striatus, Smaragdia viridis, rissoids ( $7 \mathrm{spp}$.), some species of opisthobranchs (e.g. Hancockia uncinata, Doto sp.) that feed on epibionts located on leaves of Z. marina and Mitrella minor that feeds on the abundant egg masses located on the leaf stratum;

(4) epibionts and ectoparasites (EP, Fig. 4), including mostly gastropods from families Pyramidellidae $(7 \mathrm{spp}$. associated with molluscs and polychaetes), Eulimidae (6 spp. associated with echinoderms), Epitoniidae (Epitonium spp. and Cirsotrema cochlea associated with anthozoans) and Cerithiopsidae (Cerithiopsis spp. associated with poriferans), and bivalves such as Anomia ephippium and Hiatella arctica which colonise shells of other molluscs such as Jujubinus striatus and the large prosobranch Bolma rugosa.

Other categories with a lower representation includes those of species that partly bury in the sediment (SB) (e.g. Naticidae, Cassidae, Retusidae) (Fig. 4), demersal species (DE) (e.g. cephalopods), epifaunal species of hard substrates, such as rocks or 


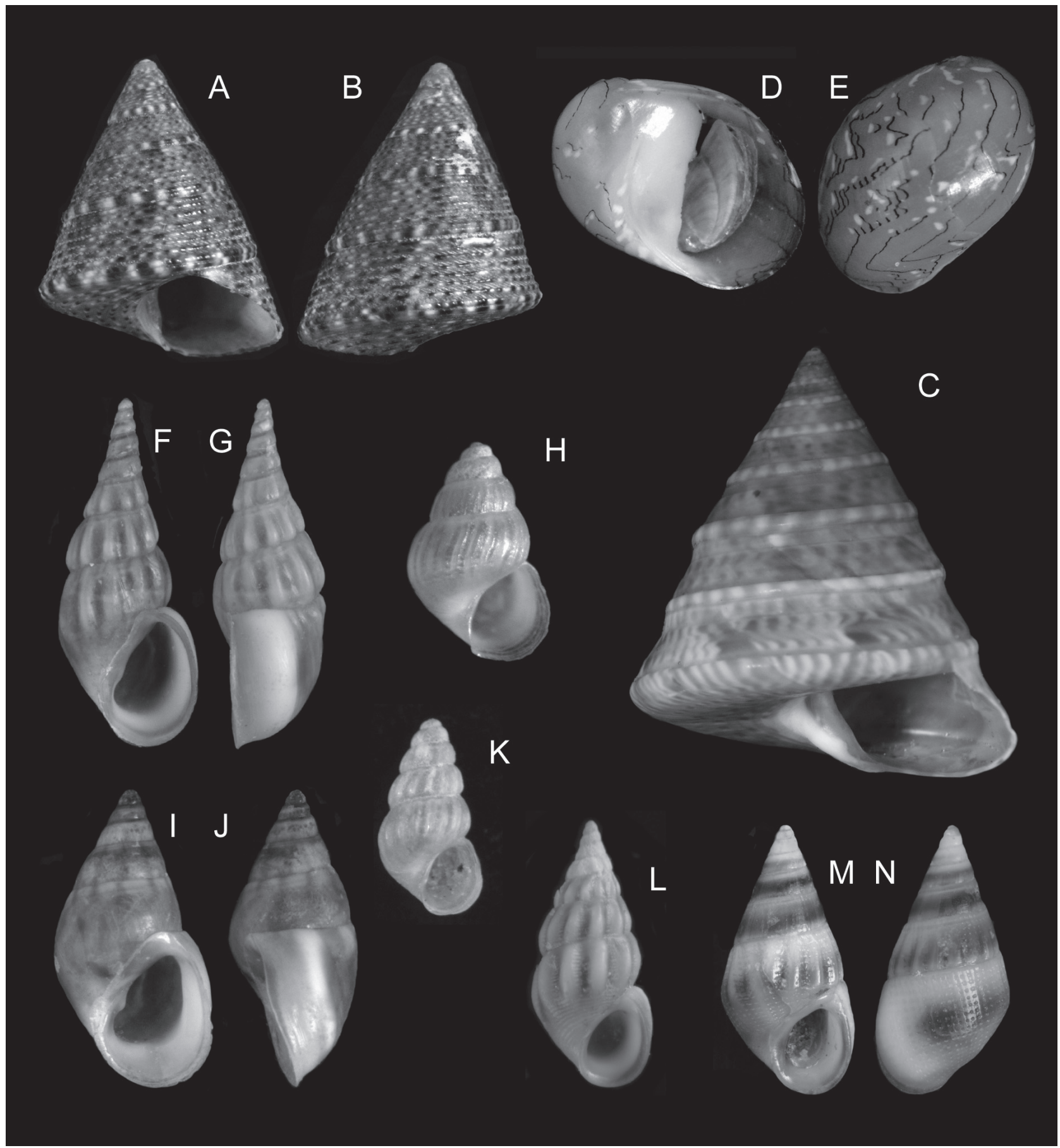

FIG. 2. - Prosobranch gastropods in the leaf stratum of Zostera marina. A, B, apertural and abapertural view of Jujubinus striatus (shell height: $7.1 \mathrm{~mm}$ ); C, apertural view of Calliostoma planatum $(9.9 \mathrm{~mm}$ ) (also inhabiting the sediment); D, E, apertural and abapertural view of Smaragdia viridis $(5.9 \mathrm{~mm}) ; \mathrm{F}, \mathrm{G}$, apertural and lateral view of Rissoa membranacea $(6.8 \mathrm{~mm}) ; \mathrm{H}$, apertural view of Pusillina inconspicua $(3.1 \mathrm{~mm}) ; \mathrm{I}, \mathrm{J}$, apertural and lateral view of Rissoa monodonta $(4.8 \mathrm{~mm}) ; \mathrm{K}$, apertural view of Pusillina phillippi $(2.1 \mathrm{~mm}) ; \mathrm{L}$, apertural view of Rissoa guerinii (4.2 mm); M, N, apertural and abapertural view of Rissoa violacea $(4.4 \mathrm{~mm})$.

shells (HE) (e.g. chitons, Gibbula spp., Lepidochitona cinerea, Williamia gussonii) and infaunal species of hard substrates (HI) (e.g. Gastrochaena dubia and Irus irus in rocks and Xylophaga praestans in wood). The two last categories mostly contain accidental species in the eelgrass bed (low \% F values).
Regarding trophic groups (Tables 2 and 3), the largest numbers of species are found in the following categories:

(1) filter feeders (F), including mostly bivalves such as venerids (9 spp.), mytilids (6 spp.), cardids (6 spp.) and pectinids (4 spp.) and a few gastropods 


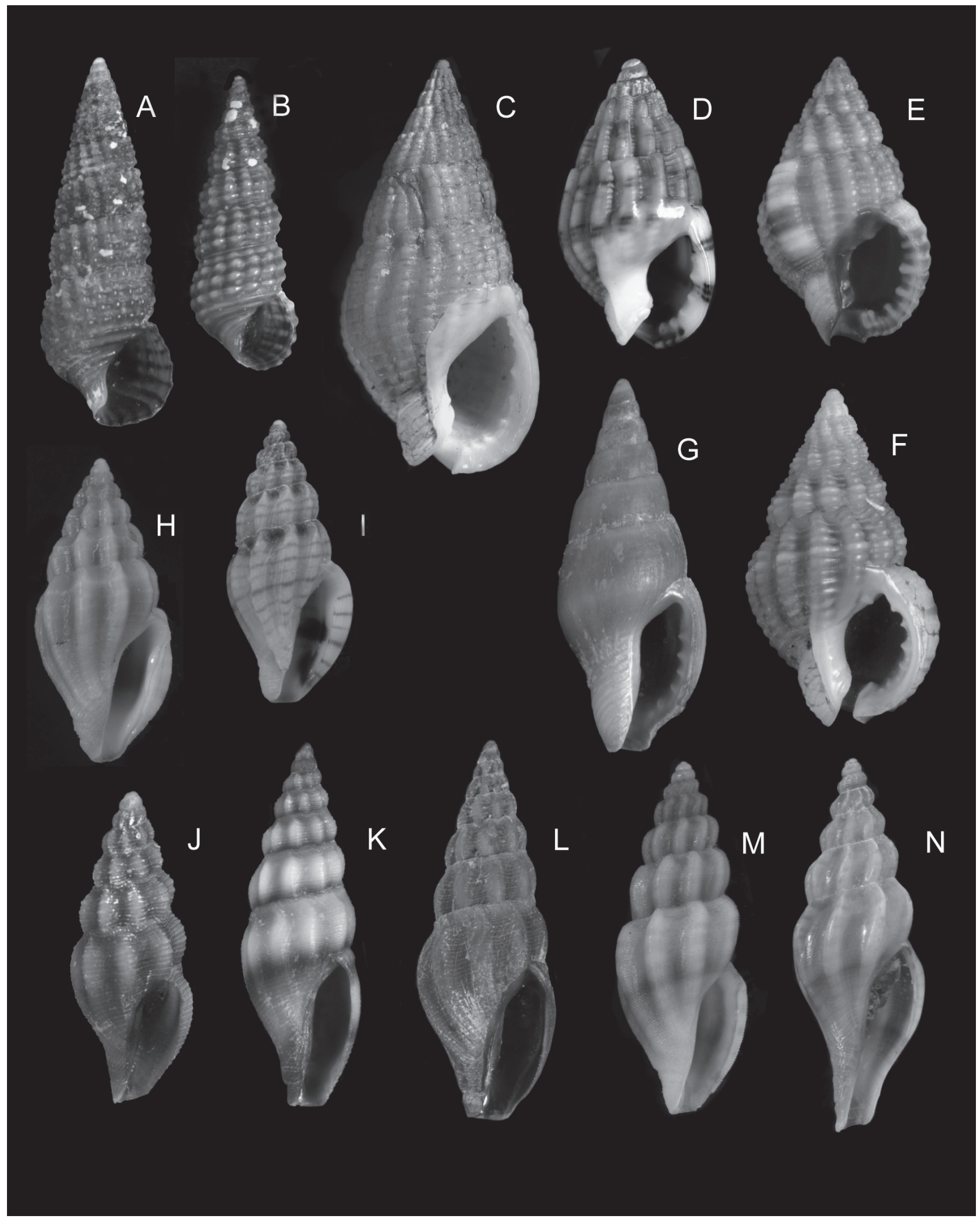

FIG. 3. - Prosobranch gastropods of the sediment and the leaf stratum in Zostera marina. Apertural views of: A, Bittium latreillii (Shell height: $7.4 \mathrm{~mm}) ; \mathrm{B}$, Bittium reticulatum $(3.8 \mathrm{~mm})$; C, Nassarius reticulatus $(12.2 \mathrm{~mm})$; D, Nassarius cuvierii $(7.7 \mathrm{~mm}) ; \mathrm{E}$, Nassarius pygmaeus $(8.7 \mathrm{~mm}) ; \mathrm{F}$, Nassarius incrassatus $(10.2 \mathrm{~mm}) ; \mathrm{G}$, Mitrella minor $(9.2 \mathrm{~mm}) ; \mathrm{H}$, Mangelia unifasciata $(8.3 \mathrm{~mm}) ; \mathrm{I}$, Mangelia paciniana $(6.1 \mathrm{~mm}) ; \mathrm{J}$, Bela sp. $1(5.8 \mathrm{~mm}) ; \mathrm{K}$, Bela laevigata $(9.9 \mathrm{~mm}) ; \mathrm{L}$, Bela nebula $(8.5 \mathrm{~mm}) ; \mathrm{M}$, Bela costulata $(7.7 \mathrm{~mm}) ; \mathrm{N}$, Mangelia attenuata $(8.1 \mathrm{~mm})$. 


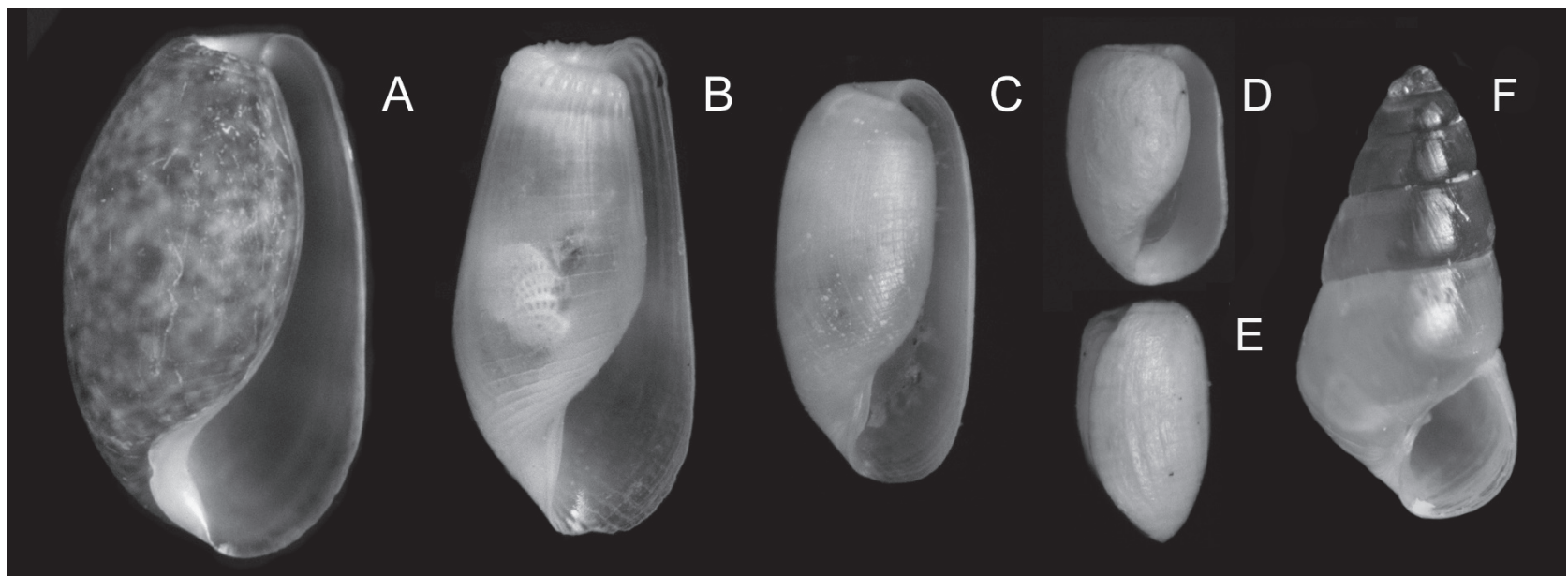

FIG. 4. - Opistobranch and Pyramidellid gastropods of Zostera marina. A, apertural view of Bulla striata (Shell height: $12 \mathrm{~mm}$ ); B, apertural view of Pyrunculus hoernesii ( $2.2 \mathrm{~mm})$, with a benthic foraminifer visible by transparency; C, apertural view of Cylichnina crossei (1.8 mm);

D, E, apertural and lateral view of Retusa minutissima $(0.9 \mathrm{~mm}) ; \mathrm{F}$, apertural view of Odostomia turrita $(1.7 \mathrm{~mm})$.

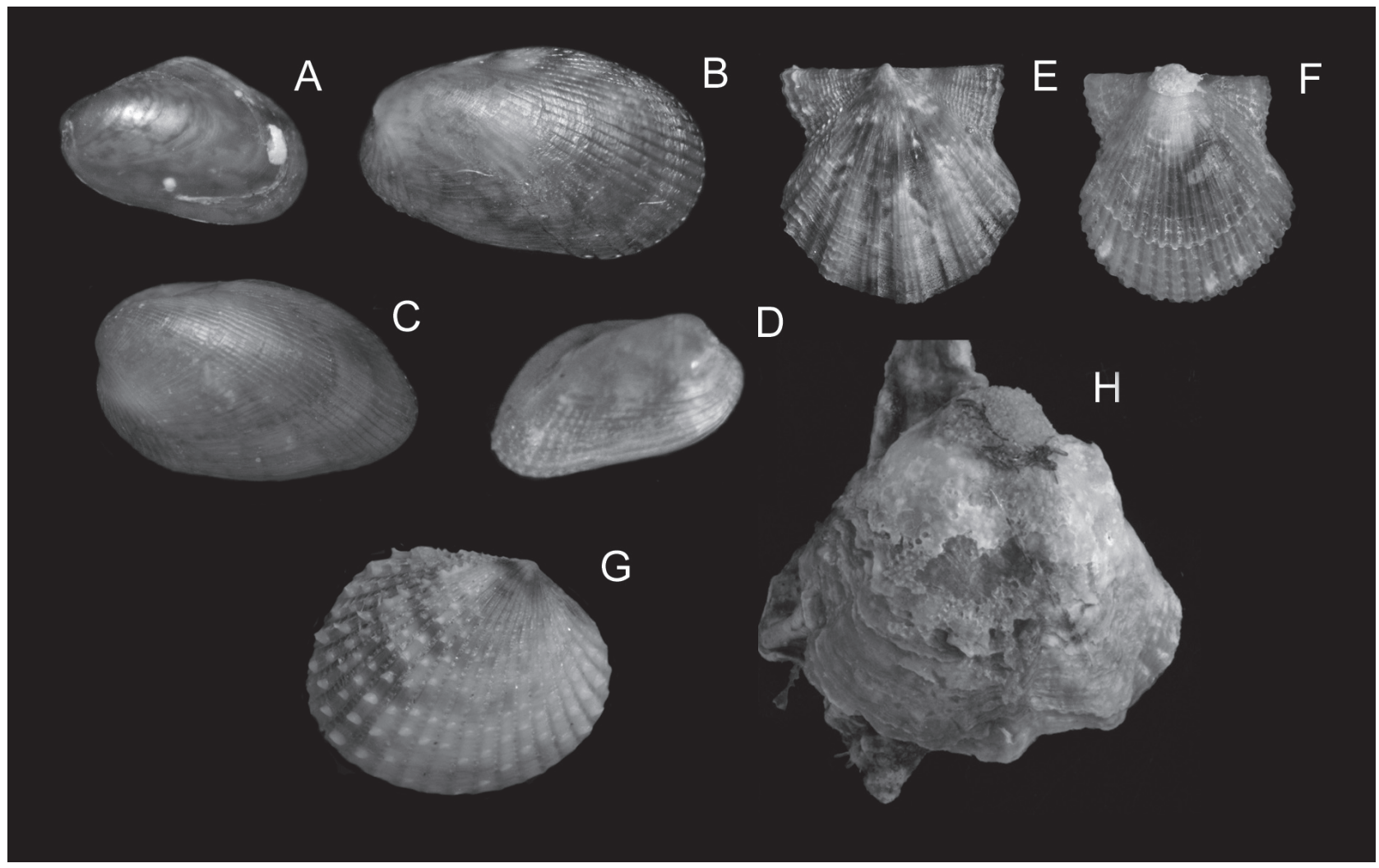

FIG. 5. - Epifaunal bivalves of Zostera marina. Outside view of: A, left valve of Mytilaster minimus (Shell length: $2.1 \mathrm{~mm}$ ); B, left valve of Musculus costulatus (3.6 mm); C, left valve of Modiolarca subpicta $(3.4 \mathrm{~mm}) ; \mathrm{D}$, right valve of Gregariella semigranata $(0.9 \mathrm{~mm})$; E, left valve of Chlamys flexuosa (4 mm); F, left valve of Chlamys opercularis $(3 \mathrm{~mm})$; G, right valve of Parvicardium scriptum (3.8 mm); H, left valve of Anomia ephippium (Maximum diameter: $4.1 \mathrm{~mm}$ ) on Ocenebra erinacea.

such as Turritella turbona, Calyptraea chinensis and Crepidula unguiformis;

(2) carnivores (C), such as gastropods from the families Conidae (11 spp.), Naticidae (3 spp.) and Muricidae (3 spp.), some opisthobranchs (10 spp.) and cephalopods (4 spp.);

(3) ectoparasites (E) represented only by gastro- pods such as pyramidellids, that live and feed on other molluscs or on sedentary polychaetes, eulimids that feed on echinoderms (e.g. Crinophtheiros sp. on crinoids, Melanella spp. on holothurians and Vitreolina philippi on echinoids), epitoniids feeding on sea anemones, Cerithiopsis spp. and the triphorids feeding on poriferans, and some opisthobranchs (e.g. 


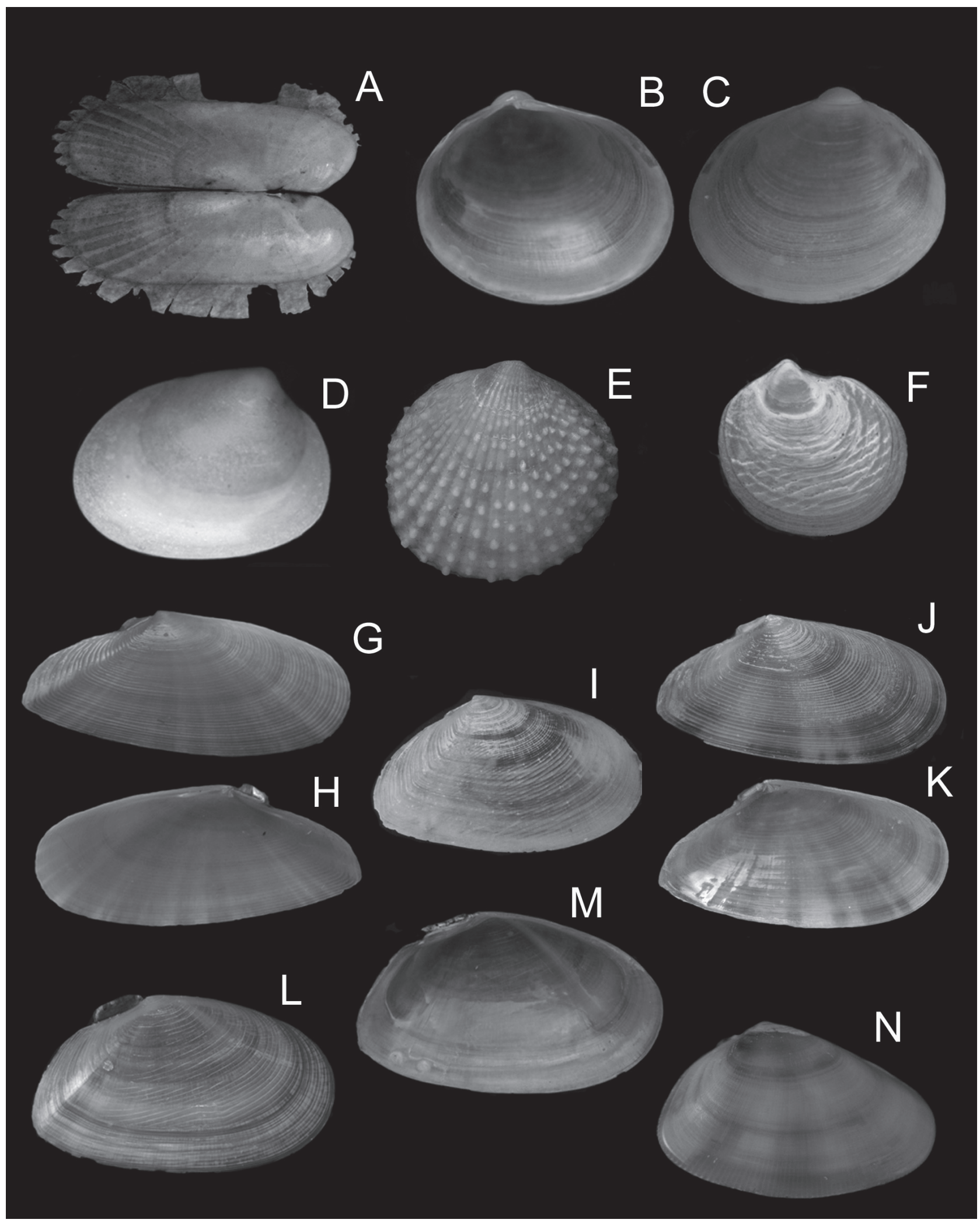

FIG. 6. - Infaunal bivalves of Zostera marina. A, outside view of Solemya togata (shell length: $6.3 \mathrm{~mm}$ ); B,C, inside and outside view of left valve of Scacchia oblonga $(2.9 \mathrm{~mm})$; D, outside view of left valve of Kurtiella bidentata $(1.7 \mathrm{~mm})$; E, outside view of right valve of Papillicardium papillosum $(5 \mathrm{~mm}) ; \mathrm{F}$, outside view of right valve of Lucinella divaricata $(1.6 \mathrm{~mm}) ; \mathrm{G}, \mathrm{H}$, outside and inside views of right valve of Tellina pulchella $(15 \mathrm{~mm})$; I, outside view of right valve of T. fabula $(8.5 \mathrm{~mm})$; J, K, outside view of right valve and inside view of left valve of $T$. distorta $(7.5 \mathrm{~mm})$; L, M, outside view of right valve and inside view of left valve of $T$. compressa (19 mm); N, outside view of right valve of a juvenile of Donax venustus (4 mm). 


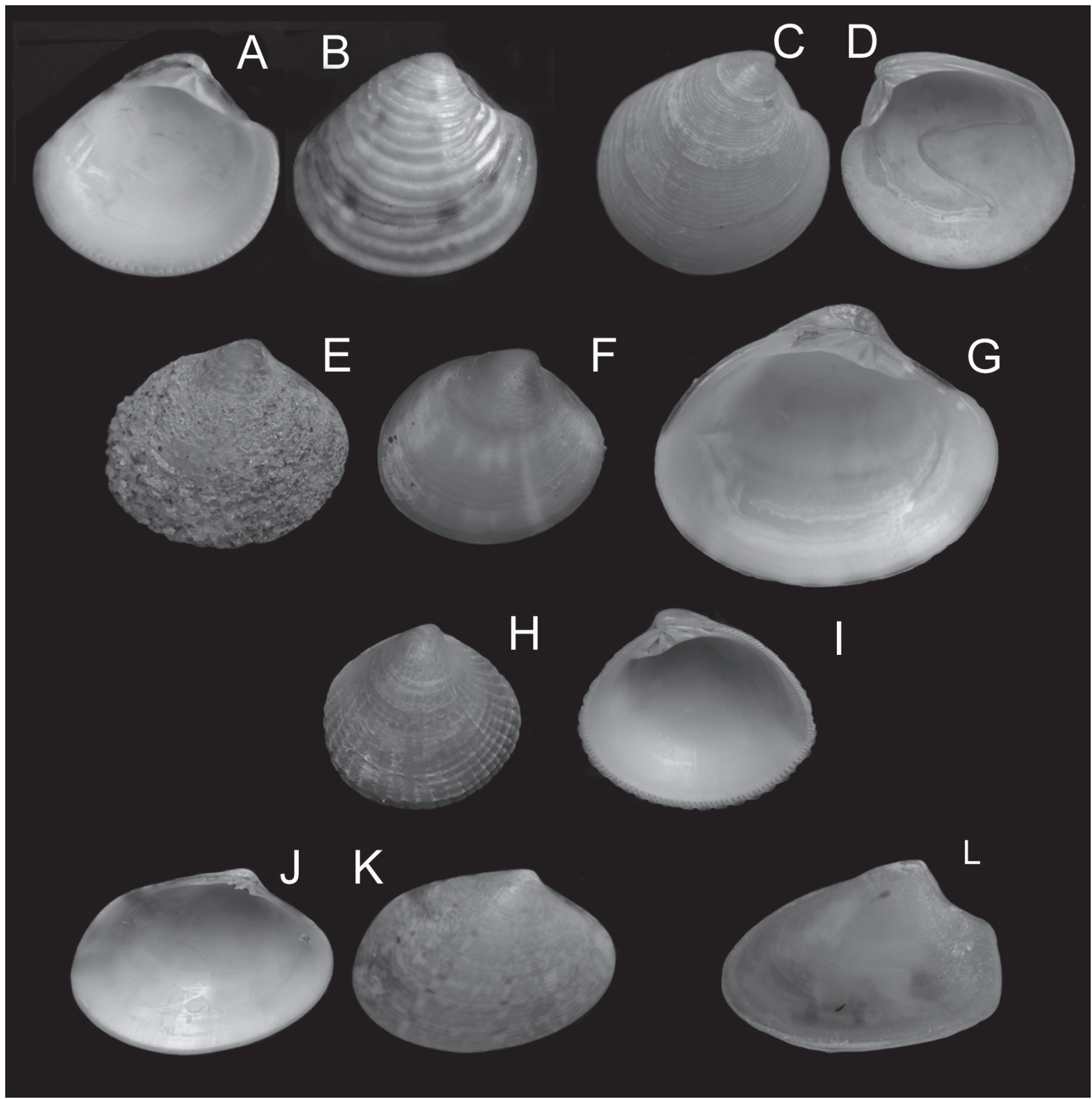

FIG. 7. - Infaunal bivalves of Zostera marina. A, B, inside view of left valve and outside view of right valve of C. gallina (shell length: $7.1 \mathrm{~mm}$ ); $\mathrm{C}, \mathrm{D}$, outside and inside view of right valve of D. lupinus $(4 \mathrm{~mm})$; E, outside view of right valve of juvenile Pitar rudis $(3.5 \mathrm{~mm})$ showing the characteristic periostracum with sand grains; $\mathrm{F}$, the same specimen without periostracum; $\mathrm{G}$, inside view of left valve of $P$. rudis $(11 \mathrm{~mm}) ; \mathrm{H}$, outside view of right valve of juvenile Timoclea ovata $(2 \mathrm{~mm})$; I, inside view of right valve of $T$. ovata $(7.5 \mathrm{~mm})$; J, K, inside view of left valve and outside view of right valve of Venerupis aurea $(11.2 \mathrm{~mm})$; L, outside view of left valve of juvenile Thracia villosiuscula $(2 \mathrm{~mm})$.

Trapania cf. lineata) feeding on bryozoans;

(4) microalgal grazers (MG), mainly including species linked to the leaf stratum of $Z$. marina such as Jujubinus striatus, Rissoa spp. and Bittium spp. and feeding on the periphyton. Most species which dominate the epifauna belong to this category (see Table 2).

Other categories with a lower representation of species are the deposit feeders (D) (e.g. tellinids,
Gari spp.), macroalgae herbivores (AG) (e.g. Tricolia spp., Bolma rugosa, Barleia unifasciata, Haminoea hydatis, Aplysia spp. and Williamia gussonii), scavengers (SC) (e.g. Nassarius spp.) and symbiontbearing species (SB) (e.g. Solemya togata, Lucinella divaricata and Xylophaga praestans). It is noteworthy that the scavengers and deposit feeders are few species but achieve high numbers in the counts of individuals (see Table 2). An interesting trophic fea- 
TABLE 3. - Number of species (N) and percentage (\%) of each category for preferred micro-habitat, feeding guild and biogeographical distribution of the molluscan fauna associated with Zostera marina beds in southern Spain. UN: unknown.

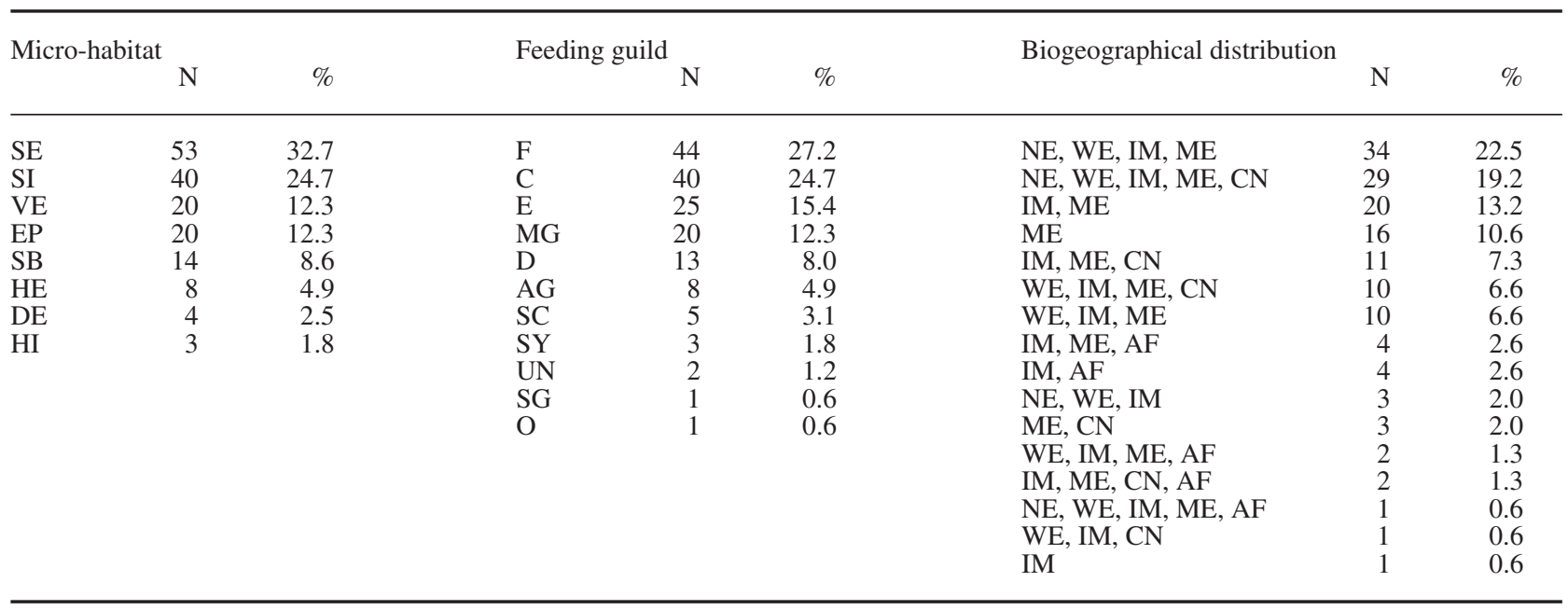

ture is the presence of one species (e.g. Mitrella minor) that feeds on egg masses and another one (e.g. Smaragdia viridis) that feeds on eelgrass epidermal tissues.

\section{Biogeographical distribution}

The species of molluscs inhabiting the studied eelgrass beds display contrasting biogeographic distributional patterns, with the Alboran Sea being their confluence area (Tables 2 and 3). According to the presence and absence on the different geographical sectors of 151 species (11 species excluded from the analysis due to poorly known distribution), the following chorotypes were obtained:

(1) species with a distribution that includes northern and western Europe, the Ibero-Moroccan Gulf and the Mediterranean Sea (NE, WE, IM, ME), including dominant gastropods associated with the leaf stratum (e.g. Jujubinus striatus, Bittium reticulatum, Pusillina inconspicua) or the sediment (e.g. Nassarius pygmaeus, Bela costulata, Euspira pulchella), ectoparasites (Odostomia turrita), infaunal bivalves (e.g. Tellina fabula, Kurtiella bidentata) and cephalopods (e.g. Sepia officinalis, Sepietta oweniana);

(2) species with a similar distributional range but also present in the Canary Islands (NE, WE, IM, ME, CN), such as the epifaunal gastropods Tricolia pullus and Nassarius incrassatus, the epifaunal bivalves Anomia ephippium, Modiolarca subpicta, Musculus costulatus and Hiatella arctica and the infaunal bivalves Dosinia lupinus, Venerupis aurea and Spisula subtruncata;
(3) species distributed along the Mediterranean Sea and the Ibero-Moroccan gulf (IM, ME) but not further in the Atlantic, such as the gastropods Mitrella minor, Tricolia tenuis, Nassarius cuvierii and Mangelia unifasciata and the bivalves Chamelea gallina and Scacchia oblonga;

(4) strictly Mediterranean species (ME), such as the gastropods Rissoa monodonta and $R$. violacea (both in the leaf stratum of Z. marina) and the infaunal bivalve Tellina planata;

(5) species present in the Mediterranean Sea, the Ibero-Moroccan Gulf and the Canary Islands (IM, ME, CN), such as the gastropods Bolma rugosa and Williamia gussonii and the bivalves Tellina distorta, Donax venustus and Parvicardium scriptum;

(6) species with a similar distribution but also reaching western Europe (WE, IM, ME, CN), such as the gastropod Bittium latreillii and the bivalves Mytilaster minimus and Lucinella divaricata;

(7) species distributed along western Europe, the Ibero-Moroccan Gulf and the Mediterranean Sea (WE, IM, ME), such as the gastropods Nassarius reticulatus and Aplysia punctata and the bivalve Thracia villosiuscula.

Other categories were represented by a lower number of species (fewer than 5 species per category) (Table 3 ).

Most species occurring in these eelgrass beds have a wide biogeographic distributional range, being present in 4 (48 spp.) or 5 (30 spp.) geographical sectors (Table 2). The faunistic composition is dominated by species which occur both along the Atlantic coasts of Europe and in the Mediterranean Sea (63 spp., Fig. 8). The species only occurring 
Bray-Curtis Similarity Index (\%)

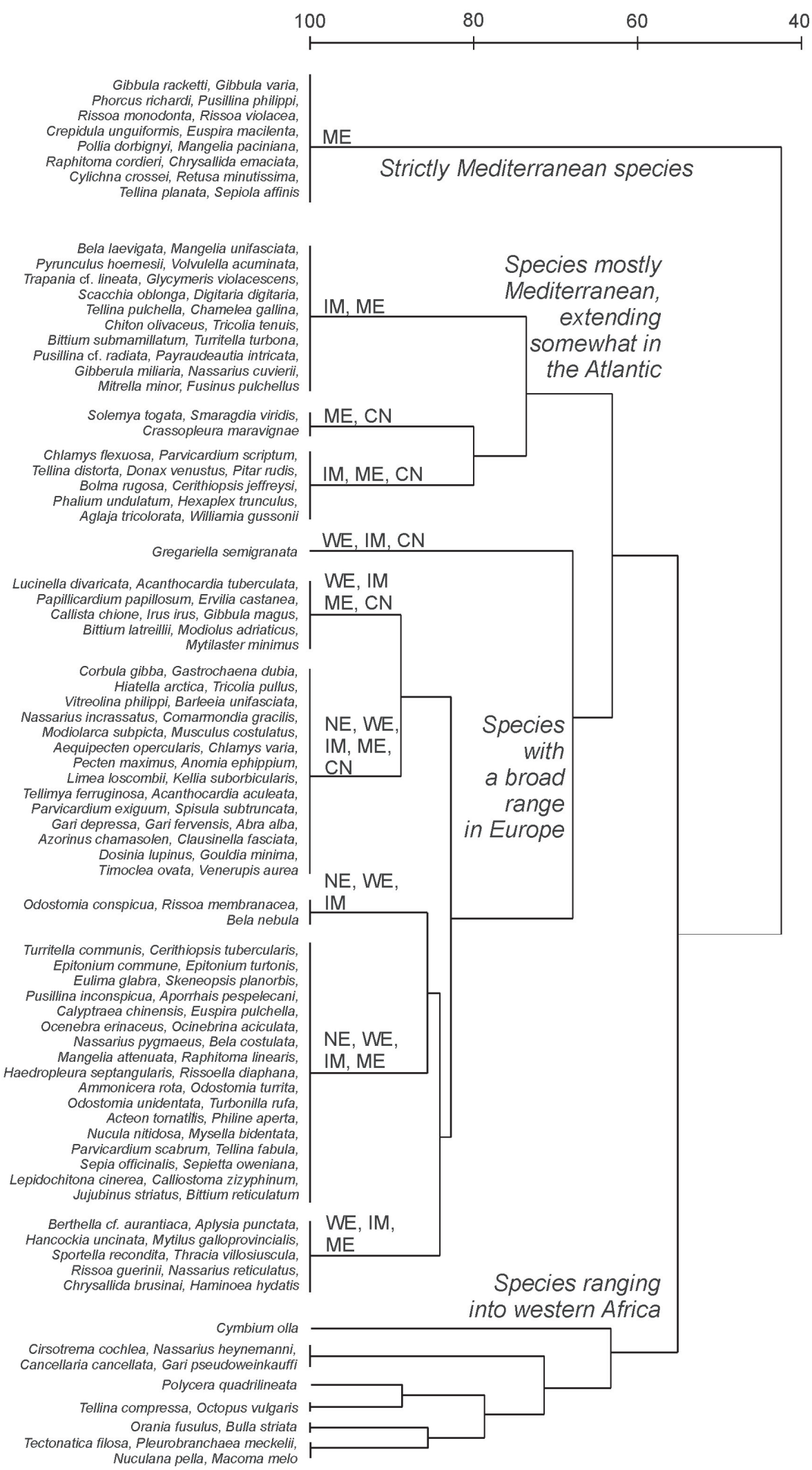

FIG. 8. - Cluster displaying faunistic groupings in relation to their presence in different geographical sectors using qualitative data (presence/ absence) and the Bray-Curtis similarity index. Codes are listed in Table 1. 
in the Mediterranean Sea (Alboran Sea excluded) represent a low number (16 spp.), as do those that are present only in the Mediterranean and in the Ibero-Moroccan Gulf (20 spp.). Only 3 species are exclusive to the Atlantic Ocean (North of Morocco) and their presence in the Mediterranean Sea is restricted to the Alboran Sea. Among these, Rissoa membranacea is a common component of eelgrass beds and adjusts to the boundary of this seagrass species in Europe. The influence of western African fauna (13 spp.) is low in comparison with the 67 species that reach the coasts of northern Europe (e.g. Scandinavia).

\section{DISCUSSION}

From a taxonomic perspective, 162 species were found living in Zostera marina beds in the Alboran Sea. This high number of species may be related to (1) the sampling methodology; (2) the presence of different micro-habitats within the eelgrass bed, (3) the availability of different food sources and (4) the biogeographical location of the studied eelgrass beds.

\section{Sampling methods}

The present faunistic list was obtained using two different sampling methodologies at different spatial scales $\left(222 \mathrm{~m}^{2}\right.$ sampled with the Agassiz trawl vs. $0.06 \mathrm{~m}^{2}$ sampled with the quadrates), and taking into account the variability throughout an annual cycle and the diurnal and nocturnal cycles. The temporal and diel variability of the molluscan assemblages in these eelgrass beds have been previously presented in Arroyo et al. (2006), Rueda and Salas (2008) and Rueda et al. (2008b) and will not be discussed further here.

Generally, a bias towards different species (e.g. epifaunal vs. infaunal, abundant vs. those occurring at low density) occurs when one is using different sampling methodologies in the same type of habitat and sampling site. In fact, a total of 23 species were only found in samples collected with the Agassiz trawl (sampling area $222 \mathrm{~m}^{2}$ ), and not in those samples collected with quadrates. Among those species, there are some that generally show a higher mobility (e.g. cephalopods) or are scarce within the eelgrass bed or in other types of habitats, such as the large-sized Bolma rugosa,
Phalium undulatum, Hexaplex trunculus, Cymbium olla, and Aplysia spp., and also Tricolia tenuis, Cerithiopsidae, Pyramidellidae, among others (Table 2). On the other hand, a total of 28 species were only found in samples collected with the quadrates method (sampling area $0.062 \mathrm{~m}^{2}$ ) and not in samples collected with the Agassiz trawl, which covered a much larger sampling area. Some of these species included small gastropods which bury in the sediment, such as Cylichnina crossei, and especially infaunal bivalves ( $21 \mathrm{spp}$.), which are very frequent (e.g. Solemya togata) and/or abundant (e.g. Lucinella divaricata, Dosinia lupinus) in this habitat and may also become the dominant species of eelgrass beds (e.g. Tellina distorta, see Table 2). In this case, a methodology based on a combination of different sampling techniques is necessary in studies on the composition and structure of the molluscan fauna associated with seagrass beds, such as those of $Z$. marina. This is not a common feature in previous studies on the fauna of eelgrass beds (Hily and Bouteille, 1999), and it generally resulted in incomplete faunistic lists or a bias towards epifaunal (Fredriksen et al., 2005; Arroyo et al., 2006; Rueda et al., 2008b) or infaunal species (Jacobs et al., 1983; Currás et al., 1993; Çinar et al., 1998; Sfriso et al., 2001).

Sampling throughout an annual cycle gave the possibility of collecting some species that may be abundant in certain seasons of the year. On a different time scale, the collection of diurnal and nocturnal samples could record those species that hide in the sediment or amongst the rhizomes during daytime (e.g. some trochids, opistobranchs) or those that generally come to the eelgrass bed at night time (e.g. cephalopods) (Templado, 1982; Mattila et al., 1999; Rueda et al., 2008b). It is important to mention that sampling was done in different years using different techniques, so variability of results between techniques could be partly due to this time-related factor. Nevertheless, similar temporal trends of the assemblages were recorded with the different sampling techniques and in different years (Arroyo et al., 2006; Rueda et al., 2008b; Rueda and Salas, 2008).

\section{Micro-habitats}

The high number of species found in this study reflects the amount of available micro-habitats that can be colonised by molluscs in comparison with 
the adjacent unvegetated areas (Currás et al., 1993; Frost et al., 1999). The eelgrass bed generally includes shoots, fine sediment and other organisms on which molluscs may live and become a dominant group (Hemminga and Duarte, 2000).

A total of 20 species of gastropods were highly associated with the leaf stratum of Z. marina, as found in other seagrass beds such as those of Posidonia oceanica (Templado, 1982; Hergueta, 1996). Some of these species are dominant or frequent throughout the year, such as Jujubinus striatus, Rissoa spp. and Smaragdia viridis (Arroyo et al., 2006; Rueda et al., 2008b). The eelgrass leaves may represent the main or exclusive food source for certain species, such as Smaragdia viridis (Rueda and Salas, 2007), or the place to find other food sources, such as peryphiton or epiphytes, which are of importance for other grazers belonging to the families Trochidae, Tricoliidae and Rissoidae (Mazzella and Russo, 1989; Hily et al., 2004).

In deep eelgrass beds of southern Spain, the species of epifaunal molluscs mainly associated with the sediment are more numerous (53 spp.) than those associated with the leaf stratum. The sediment covered by eelgrass is fine sand containing some mud and is rather similar in vegetated and unvegetated areas (Rueda et al., 2008a). This contrasts with P. oceanica beds in which the vegetated and unvegetated transition can be very abrupt, with the rhizomes building up reef-like formations that are equivalent to a hard substrate, and channels filled with different types of sediments and dead leaves. The smooth transition between vegetated and unvegetated areas in eelgrass beds may promote the flux of epifaunal species (e.g. Nassarius spp., Naticidae, Cylichnina spp.) from adjacent unvegetated areas in which they can also be abundant. This is also applicable to most infaunal species (40 spp.) (e.g. Tellina spp., Abra alba, Corbula gibba, Spisula subtruncata and Chamelea gallina), which are also common in unvegetated bottoms (Rueda et al., 2001; Rueda and Salas, 2003b), and occur inside the meadow if they resist the higher amounts of mud and organic matter in the sediment covered by Z. marina (Rueda et al., 2008a). In other cases, some characteristics of the sediment covered by eelgrass, such as the redox level, may benefit different infaunal species, such as Solemya togata and Lucinella divaricata, which are generally not common in unvegetated sediments.

Diversity of micro-habitats within a seagrass bed normally generates biodiversity of species such as those of molluscs, as has been found in other seagrasses from southern Spain such as Posidonia oceanica (Templado, 1982; Hergueta, 1996), Cymodocea nodosa (Templado, 1982; Ballesteros et al., 2004) and Zostera noltii (Templado, 1982; Pérez Lloréns, 2004). In P. oceanica, the presence of different types of micro-habitats is enhanced by the availability of both a hard and complex substratum (rhizomes and calcareous algae) and a leaf stratum, resulting in high numbers of associated mollusc species (around 200 species) (Templado, 1982; Hergueta, 1996). In Cymodocea nodosa, the microhabitats available are somehow similar to those of Z. marina, but the faunistic lists have generally resulted in lower numbers of species, between 30 and 50 spp. (Templado, 1982; Ballesteros et al., 2004 and studies in progress by Marina in Almería, SE Spain). This is probably because the leaf stratum of C. nodosa generally offers less leaf surface available (leaf height and width up to $40 \mathrm{~cm}$ and 2-4 $\mathrm{mm}$ and 4-5 leaves per shoot) and is therefore less complex than that of $Z$. marina (leaf height and width up to $60 \mathrm{~cm}$ and 5-6 $\mathrm{mm}$ and up to 7 leaves per shoot) (Green and Short, 2003; Urra et al., 2008). This may also result in a lower presence, in C. nodosa beds, of other faunistic groups (e.g. equinoderms, poriferans, anthozoans) that are essential for some molluscs (e.g. eulimids, triphorids, epitonids). A lower number of species (between 10 and $20 \mathrm{spp}$.) seems to be associated with Z. noltii, but in this case it is mainly due to the brackish conditions in which these beds occur (Templado, 1982; Pérez Lloréns, 2004). In southern Spain, Zostera marina beds display larger faunistic lists than other soft bottom seagrasses such as $C$. nodosa and Z. noltii. Nevertheless, $P$. oceanica beds still contain the richest molluscan assemblage, as the result of its unequalled diversity of micro-habitats.

\section{Feeding guilds}

In the studied Z. marina beds, a large number of species are carnivores (40 spp.), including predators of other molluscs (e.g. Naticidae), ectoparasites and specialised carnivores $(24 \mathrm{spp}$.) that feed on other invertebrates (e.g. Eulimidae, Pyramidellidae). This is also a reflection of the high biodiversity of other groups of invertebrates that inhabit these deep subtidal Z. marina beds of the Alboran Sea (García Raso et al., 2004). The high availability and variety of food sources for carnivores is comparable to that found in P. oceanica beds (Templado, 1984b), which attracts 
carnivores from adjacent unvegetated bottoms and macroalgal beds.

In comparison with the carnivore species, the number of species grazing on microalgae (20 spp.) and macroalgae ( $8 \mathrm{spp}$.) is low but these display the highest dominances among the epifaunal species (e.g. Jujubinus striatus, Rissoa spp., Bittium reticulatum) (Arroyo et al., 2006; Rueda et al., 2008b, see Table 2). The competition for these food sources is probably stronger than for the highly diverse food sources of carnivores, and this may promote the presence of a few dominant and efficient species of grazers, such as Jujubinus striatus (Peduzzi, 1987; Hily et al., 2004). Nevertheless, some components of this herbivore group are also abundant in other vegetated habitats such as Caulerpa prolifera beds (Rueda and Salas, 2003a) and P. oceanica beds (Templado, 1982; Hergueta, 1996), so their dependence for eelgrass beds is questionable.

Two interesting trophic groups are otherwise represented in the Z. marina beds of southern Spain and not in other eelgrass beds along European coasts. One is the seagrass feeders, which are only represented by the neritid Smaragdia viridis (Rueda and Salas, 2007). This gastropod is distributed along the Mediterranean Sea, the Canary Islands and also in Bermuda and the Caribbean Sea, and in all cases it is strictly associated with certain species of seagrasses, feeding on their epidermal tissues. The second unusual trophic group is the egg feeder Mitrella minor, already alluded in previous works (García Raso et al., 2004, Arroyo et al., 2006). Its high frequency and dominance throughout the year are consistent with the importance of the eelgrass beds as a spawning site (Hemminga and Duarte, 2000).

In relation to the infaunal species, the filter feeders are the most diverse (42 spp.), followed by the deposit feeders (13 spp.), the latter being benefited by the high amount of organic matter present in the fine sediment covered by the eelgrass (Rueda et al., 2008a). The availability of fine sediment within the Z. marina bed favours the persistence of both trophic groups, contrary to $P$. oceanica beds where the food resource and sediment type is limited for deposit feeders and where bivalves are generally filter feeders or bearing endosymbionts (Templado, 1984a, b).

Regarding taxonomic families, and therefore trophic groups, the molluscan fauna associated with Z. marina beds in southern Spain is comparable to that found in other eelgrass beds around the northern Hemisphere. In the Atlantic coasts of North America, the dominant molluscan species belong to the families Cerithiidae, Retusidae, Nassariidae, Pyramidellidae, Columbellidae, Mytilidae, Tellinidae, Veneridae, Semelidae and Solemyidae (Marsh, 1973; 1976; Thayer et al., 1975; Mattila et al., 1999). In Japan (Pacific Ocean), the dominant epifaunal molluscs of eelgrass beds located at 2-3.5 metres depth are represented by species of the families Phasianellidae, Trochidae, Rissoidae and Nassariidae (Toyohara et al., 1999; Nakaoka et al., 2001). Therefore, in a global context, the molluscs associated with $Z$. marina beds are dominated by species of grazers on microalgae associated with the leaf stratum (e.g. Trochidae, Cerithidae, Rissoidae), infaunal deposit feeders (e.g. Tellinidae, Semelidae), epifaunal scavengers (e.g. Nassariidae), infaunal and epifaunal filter feeders (e.g. Mytilidae, Veneridae) and epifaunal carnivores (e.g. Conidae, Columbellidae).

An important question to answer is: How many of the mollusc species are strictly dependent on eelgrass? The species which may be strictly associated are to be found among grazers in the leaf stratum. These could include Jujubinus striatus, Tricolia tenuis, Smaragdia viridis, Rissoa membranacea, $R$. monodonta and $R$. violacea. However, these species were also reported on Cymodocea nodosa (Ballesteros et al., 2004), which implies that the association is not species-specific. Furthermore, Jujubinus striatus was also found in association with the foliose alga Caulerpa prolifera, although less abundant (Rueda and Salas, 2003a). Therefore, the requirements for the periphyton grazers like $J$. striatus seem to be limited to a kind of substrate, with the option to replace the preferred eelgrass with leaf-like algal fronds. Contrary to the terrestrial ecosystems, where specialised phytophagous species are commonplace, the sole specialised herbivore in the eelgrass seems to be Smaragdia viridis and even this one can feed on C. nodosa (Rueda and Salas, 2007). The remainder of the fauna registered in the eelgrass bed seems to draw from a variety of compartments of the benthic ecosystem and to take advantage of the multiple resources in the eelgrass bed in an opportunistic way, rather than depending on this plant as a food source.

\section{Biogeographical distribution}

The location of the studied eelgrass beds, between the Atlantic Ocean, the Mediterranean Sea and northern Africa, represents the confluence area for different marine species from the Mediterranean, 
Lusitanian and Mauritanian regions (Ekman, 1953). In general, there is an increase of molluscan species richness from northern to southern Europe: $380 \mathrm{spp}$. at the entrance to the English Channel (Cornet and Marche-Marchad, 1951; Marine Biological Association of the UK, 1957), 660 spp. in Galicia (northwestern Spain) (Rolán, 1983; Rolán et al., 1990) and around 1000 spp. in the Alboran Sea (655 spp., excluding those from soft bottoms, listed in Peñas et al., 2006).

The latitudinal trend in the molluscan composition of eelgrass beds is difficult to separate from the possible influence of depth because eelgrass beds of the Alboran Sea occur much deeper than in other parts of Europe. Generally, a trend for increasing number of species occurs from intertidal to subtidal seagrass beds (Hemminga and Duarte, 2000) and this may be due to the increased stability of physical parameters at deeper locations. Admittedly, the trend is not tested within a single transect in this study but, nevertheless, the number of species we registered is markedly higher than those found in shallower beds located in the Atlantic Ocean (with 5-10 dominant and highly frequent species) (Jacobs et al., 1983; Jacobs and Huisman, 1982; Currás et al., 1993; Boström and Bonsdorff, 1997; Hily and Bouteille, 1999; Fredriksen et al., 2005; Quintas, 2005) or the Mediterranean Sea (around 5-7 spp.) (Ledoyer, 1966; Mars, 1966; Çinar et al., 1998; Sfriso et al., 2001).

In estuaries and brackish waters of northern Europe, the molluscan taxocoenosis (up to $10 \mathrm{spp}$.) associated with eelgrass beds is the typical estuarine molluscan fauna with Hydrobia spp., Theodoxus fluviatilis and Littorina littorea and the bivalves Cerastoderma edule, Scrobicularia plana and Macoma balthica (Jacobs et al., 1983; Boström and Bonsdorff, 1997; 2000), the latter being a Nordic species. On the other hand, the molluscan fauna of eelgrass beds in marine coastal areas along Atlantic Europe (up to $40 \mathrm{spp}$.) is dominated by the gastropods Jujubinus striatus, Rissoa membranacea, Rissoa parva, Bittium reticulatum, Tricolia pullus, Gibbula pennanti, Nassarius reticulatus and Lacuna vincta and by bivalves such as Loripes lacteus, Abra alba and Parvicardium exiguum (Jacobs and Huisman, 1982; Currás et al., 1993; Frost et al., 1999; Hily and Bouteille, 1999; Fredriksen et al., 2005; Quintas, 2005). Among these, R. parva, G. pennanti and $L$. vincta are strictly Atlantic and the rest of the species are present in the eelgrass beds of southern Spain.
On the Mediterranean coasts of Europe, the molluscan fauna associated with eelgrass beds (around 20 spp.) has been poorly studied and includes the gastropods Bittium reticulatum, Rissoa grossa, Rissoa lineolata, Gibbula adansonii and bivalves such as Loripes lacteus, Parvicardium exiguum and Venerupis aurea (Ledoyer, 1966; Mars, 1966; Çinar et al., 1998; Sfrisso et al., 2001). Among these, $R$. lineolata and $G$. adansonii are endemic to the Mediterranean Sea and do not reach the Alboran Sea and $R$. grossa could represent a synonym or a geographic subspecies of $R$. membranacea.

Eelgrass beds are more common on the Atlantic coasts of Europe than in the Mediterranean Sea (Green and Short, 2003), but most molluscan species associated with the subtidal Z. marina beds of the Alboran Sea are also present in both the IberoMoroccan Gulf and the Mediterranean Sea (81.2\%), with a low representation of species that are strictly Mediterranean (10.6\%) or Atlantic (2.0\%) (Table 3 ). This means that the molluscan fauna of these Z. marina beds is essentially derived from the local fauna, which includes many widely distributed species along European coasts (Table 2). The apparent contradiction between the Atlantic main range of Zostera marina and the character of the local fauna with Mediterranean components may be in part explained if the malacofauna of eelgrass beds in Cantarrijan and Cañuelo can draw from the common Mediterranean fauna usually found associated with other common soft bottom seagrasses such as Cymodocea nodosa (Chemello et al., 1997; Sfriso et al., 2001; Ballesteros et al., 2004). Studies in progress on the epifaunal molluscan assemblage of Cymodocea nodosa from the Bay of Genoveses (Almería, SE Spain) show that $84 \%$ of the 53 listed species from $C$. nodosa are present in these Z. marina beds; $6 \%$ are strictly Mediterranean and do not enter the Alboran Sea; and the remainder are rare or accidental. Other species would be drawn from other neighbouring habitats from that area, such as macroalgae, soft bottoms or rocky shores.

Seagrass beds are declining world-wide and a further decrease is expected during this century, especially in non-developed countries (Duarte, 2002). In southern Spain, a strong decline of certain types of seagrass beds such as the Z. marina beds of this study has also been detected in the last years due to illegal trawling by fishermen, among other impacts (Rueda et al., 2008a). A better management and patrol of these types of habitats is urgently needed 
in this part of southern Europe, or we will have to say goodbye to one of the most diverse molluscan faunas and communities (García Raso et al., 2004; Reina-Hervás et al., 2006) living in eelgrass beds of the old continent.

\section{ACKNOWLEDGEMENTS}

We are grateful to María del Carmen Arroyo, Enrique García Raso, José Reina-Hervás, Pablo Marina, María Eugenia Manjón-Cabeza, Vanesa Cobos, Vanessa Díaz and María Jose Martín from the University of Malaga (Spain) for their help in the collection and processing of samples, to Antonio Pulido (Director of the MPA, Junta de Andalucía) for sampling permissions, to Michelle K. Nishiguchi from New Mexico State University (USA) for identification of cephalopod specimens and to Dr Boris Sirenko of the Russian Academy of Sciences (Russia) for identification of juvenile chiton specimens. The Spanish M.E.C. financially supported this study, DGICYT funds, Project PB97-1116 "Estudio de la macrofauna de los fondos de fanerógamas marinas, Zostera y Cymodocea, del Sur de España”.

\section{REFERENCES}

Aartsen, J. van, H.P.M.G. Menkhorst and E. Gittenberger. - 1984. The marine mollusca of the bay of Algeciras, Spain, with general notes on Mitrella, Marginellidae and Turridae. Basteria, Suppl. 2: 1-135.

Arroyo, M.C., C. Salas, J.L. Rueda and S. Gofas. - 2006. Temporal changes of mollusc populations from a Zostera marina bed in southern Spain (Alboran Sea), with biogeographic considerations. Mar. Ecol., 27: 417-430.

Ballesteros, E., J.E. García Raso, C. Salas, S. Gofas, D. Moreno and J. Templado. - 2004. Las praderas de Cymodocea nodosa. La comunidad de Cymodocea nodosa: Flora y Fauna. In: Á.A. Luque and J. Templado (eds.), Praderas y bosques marinos de Andalucía, pp. 146-153. Consejería de Medio Ambiente, Junta de Andalucía, Sevilla.

Bañares-España, E., J.C. Báez, M.D. Casado, C. Díaz de Rada, A. Flores-Moya and J. Rey. - 2002. Distribución y estado de las fanerógamas marinas en el Paraje natural Acantilados de MaroCerro gordo (Málaga-Granada). In: J.C. García-Gómez and C. Finlayson, (eds.), Libro de Resúmenes - XII Simposio Ibérico del Bentos Marino, pp. 30-31. Impresur, Algeciras.

Beesley, P.L., G.J.B. Ross and A. Wells. - 1998. Mollusca: The Southern Synthesis. Fauna of Australia. Vol. 5. CSIRO Publishing, Melbourne.

Boström, C. and E. Bonsdorff. - 1997. Community structure and spatial variation of benthic invertebrates associated with Zostera marina (L.) beds in the northern Baltic Sea. J. Sea Res., 37: 153-166.

Boström, C. and E. Bonsdorff. - 2000. Zoobenthic community establishment and habitat complexity - the importance of seagrass shoot-density, morphology and physical disturbance for faunal recruitment. Mar. Ecol. Prog. Ser., 205: 123-138.

Bouchet, P., F. Danrigal and C. Huyghens. - 1979. Seashells of Western Europe. American Malacologists Inc., Florida.
Bray, R.J. and J.T. Curtis. - 1957. An ordination of the uplands forest communities of southern Wisconsin. Ecol. Monogr., 27: 325-347.

Briggs, J.C. - 1974. Marine Zoogeography. Mc Graw Hill, New York.

Chemello, R., G. Scotti and S. Riggio. - 1997. The molluscan assemblage of the leaf stratum in a Cymodocea nodosa bed of a marine coastal lagoon. Boll. Malacol., 33: 69-76.

Clarke, K.R. and R.M. Warwick. - 1994. Change in marine communities: an approach to statistical analysis and interpretation. Plymouth Marine Laboratory, Bourne Press Ltd., Bournemouth.

Cornet, R. and I. Marche-Marchad. - 1951. Inventaire de la faune marine de Roscoff, Mollusques. Trav. Stat. Biol. Roscoff, Suppl. 5: $1-80$.

Currás, A., A. Sánchez-Mata and J. Mora. - 1993. Estudio comparativo de la macrofauna bentonica de un fondo de Zostera marina y un fondo arenoso libre de cubierta vegetal. Cah. Biol. Mar., 35: 91-112.

Çinar, M.E., Z. Ergen, B. Ozturk and F. Kirkim. - 1998. Seasonal analysis of zoobenthos associated with a Zostera marina $\mathrm{L}$. bed in Gulbahce Bay (Aegean Sea, Turkey). P.S.Z.N.I.: Mar. Ecol., 19: $147-162$.

Duarte, C.M. - 2002. The future of seagrass meadows. Env. Cons., 29: 192-206.

Ekman, S. - 1953. Zoogeography of the sea. Sidgwick and Jackson, London.

Fredriksen, S., C. Hartvig and B.A. Sæthre. - 2005. Species richness in macroalgae and macrofauna assemblages on Fucus serratus L. (Phaeophyceae) and Zostera marina L. (Angiospermae) in Skagerrak, Norway. Mar. Biol. Res., 1: 2-19.

Fretter, V. and A. Graham. - 1962. British Prosobranch Molluscs. Their funcional Anatomy and Ecology. Bartholomew Press, Dorking.

Frost, M.T., A.A. Rowden and M.J. Attrill. - 1999. Effect of habitat fragmentation on the macroinvertebrate infaunal communities associated with the seagrass Zostera marina L. Aquat. Conserv., 9: 255-263.

Gambi, M.C., M. Lorenti, G.F. Russo, M.B. Scipione and V. Zupo. - 1992. Depth and seasonal distribution of some groups of the vagile fauna of the Posidonia oceanica leaf stratum: Structural and trophic analyses. P.S.Z.N.I: Mar. Ecol., 13: 17-39.

García Raso, J.E., C. Salas, S. Gofas, A. Barrajón and J.E. GarcíaMuñoz. - 2004. Las praderas de Zostera marina. La comunidad de Zostera marina: Flora y Fauna. In: Á.A. Luque and J. Templado (eds.), Praderas y bosques marinos de Andalucía, pp. 162-172. Consejería de Medio Ambiente, Junta de Andalucía, Sevilla.

Gofas, S. and A. Zenetos. - 2003. Exotic molluscs in the Mediterranean basin: Current status and perspectives. Oceanogr. Mar. Biol., 41: 237-277.

Gómez-Rodríguez, R. and J.M. Pérez-Sánchez. - 1997. Moluscos Bivalvos de Canarias. Ediciones del Cabildo Insular de Gran Canaria, Gran Canaria.

Graham, A. - 1971. British Prosobranch and other operculate gastropod molluscs. Keys and notes for the identification of the species. Academic Press, London.

Green, E.P. and F.T. Short. - 2003. World Atlas of Seagrasses. University of California Press, California.

Hansson, H.G. - 1998. NEAT (North East Atlantic Taxa): Scandinavian marine Mollusca Check-List. Internet Ed., Aug. 1998 [http://www.tmbl.gu.se].

Hayward, P.J. and J.S. Ryland. - 1995. Handbook of the Marine Fauna of North-West Europe. Oxford University Press, Oxford.

Hemminga, M.A. and C.M. Duarte. - 2000. Seagrass Ecology. Cambridge University Press, Cambridge.

Hergueta, E. - 1996. Estudio de las taxocenosis malacológicas asociadas a concrecionamientos de Mesophyllum lichenoides (Ellis) Lemoine y a una pradera de Posidonia oceanica (Linnaeus) Delile del litoral almeriense. Ph.D. thesis, Univ. Málaga.

Hily, C. and M. Bouteille. - 1999. Modifications of the specific diversity and feeding guilds in an intertidal sediment colonized by an eelgrass meadow (Zostera marina) (Brittany, France). $C$. R. Acad. Sci. III-Vie, 322: 1121-1131.

Hily, C., S. Connan, C. Raffin and S. Willey-Echeverria. - 2004. In vitro experimental assessment of the grazing pressure of two 
gastropods on Zostera marina L. epiphytic algae. Aquat. Bot., 78: $183-195$.

Høisæter, T. - 1985. An annotated check-list of Marine molluscs of the Norwegian coast and adjacent waters. Sarsia, 71: 73-145.

Jacobs, R.P.W.M. and W.H.T. Huisman. - 1982. Macrobenthos of some Zostera beds in the vicinity of Roscoff (France) with special reference to relations with community structure and environmental factors. P. K. Ned. Akad. Wetensc., 85: 335-356.

Jacobs, R.P.W.M., H.H. Hegger and A. Ras-Willems. - 1983. Seasonal variations in the structure of a Zostera community on tidal flats in the SW Netherlands, with special reference to the benthic fauna. P. K. Ned. Akad. Wetensc., 86: 347-375.

Kohn, A.J. - 1983. Feeding biology of Gastropods. In: A.S.M. Saleuddin and K.M. Wilbur (eds.), The Mollusca, vol. 5 Physiol ogy (part II), Academic Press, New York.

Ledoyer, M. - 1962. Etude de la faune vagile des herbiers superficiels de Zosteracées et de quelques biotopes d'algues littorales. Rec. Trav. St. Mar. End., 25: 117-235.

Ledoyer, M. - 1966. Écologie de la faune vagile des biotopes méditerranéens accessibles en scaphandre autonome. II. Donnés analytiques sur les herbiers de phanérogames (1). Rec. Trav. St. Mar. End., 41: 135-165.

Longhurst, A. - 1998. Ecological Geography of the Sea. Academic Press, London.

Luque, Á.A. - 1984. Contribución al conocimiento de los moluscos gasterópodos de las costas de Málaga y Granada. Ph.D. thesis, Univ. Complutense de Madrid.

Luque, Á.A. - 1986. El genero Mitrella Risso, 1826 (Gastropoda, Columbellidae) en las costas Ibéricas. Boll. Malacol., 22: 223-244.

Luque, Á.A. and J. Templado. - 2004. Praderas y bosques marinos de Andalucía. Consejería de Medio Ambiente, Junta de Andalucía, Sevilla.

Marine Biological Association of the United Kingdom, 1957. Plymouth Marine Fauna. Marine Biological Association of the United Kingdom, Plymouth.

Mars, P. - 1966. Recherches sur quelques étangs du litoral méditerranéen francais et sur leurs faunes malacologiques. Vie Milieu, 20: $1-359$.

Marsh, G.A. - 1973. The Zostera epifaunal community in the York River, Virginia. Chesapeake Sci., 14: 87-97.

Marsh, G.A. - 1976. Ecology of the gastropod epifauna of eelgrass in a Virginia estuary. Chesapeake Sci., 17: 182-187.

Mattila, J., G. Chaplin, M.R. Eilers, K.L. Heck, J.P. O'Neal and J.F. Valentine. - 1999. Spatial and diurnal distribution of invertebrate and fish fauna of a Zostera marina bed and nearby unvegetated sediments in Damariscotta River, Maine (USA). $J$. Sea Res., 41: 321-332.

Mazzella, L. and G.F. Russo. - 1989. Grazing effect of two Gibbula species (Mollusca, Archaeogastropoda) on the epiphytic community of Posidonia oceanica leaves. Aquat. Bot., 35 357-373.

Moreno, D. and J. Guirado. - 2003. Nuevos datos sobre la distribución de las fanerógamas marinas en las provincias de Almería y Granada (SE España). Acta Bot. Malacitana, 28 105-120.

Morton, J.E. - 1967. Molluscs. Hutchinson University Library, London.

Nakaoka, M., T. Toyohara and M. Matsumasa. - 2001. Seasonal and between-substrate variation in mobile epifaunal community in a multispecific seagrass bed of Otsuchi Bay, Japan. P.S.Z.N.I. Mar. Ecol., 22: 379-395.

Nobre, A. - 1940. Fauna malacológica de Portugal - I, Moluscos marinhos e das águas salobras. Barcelos: Companhia Editora do Minho.

Nordsieck, F. and F. García-Talavera. - 1979. Moluscos marinos de Canarias y Madeira (Gastropoda). Aula de Cultura de Tenerife.

Pallary, P. - 1920. Exploration scientifique du Maroc organisée par la Société de Géographie de Paris et continuée par la Société des Sciences Naturelles du Maroc. Deuxième fascicule. Malacologie (1912). Larose, Rabat/ Paris.

Peduzzi, P. - 1987. Dietary preferences and carbon absorption by two grazing gastropods Gibbula umbilicaris (Linné) and Jujubinus striatus (Linné). P.S.Z.N.I. Mar. Ecol., 8: 359-370

Peñas, A., E. Rolán, Á.A. Luque, J. Templado, D. Moreno, F. Rubio, C. Salas, A. Sierra and S. Gofas. -2006 . Moluscos marinos de la isla de Alborán. Iberus, 24: 23-151.

Pérez Lloréns, J.L. - 2004. Las praderas de Zostera noltii. In: Á.A. Luque and J. Templado (eds.), Praderas y bosques marinos de Andalucía, pp. 173-179. Consejería de Medio Ambiente. Junta de Andalucía, Sevilla.

Quintas, P. - 2005. Distribución espacial y temporal de los molus$\cos$ y anélidos poliquetos asociados a las praderas de Zostera marina L. y Zostera noltii Hornem. en la ensenada de O Grove (Galicia, España). Ph. D. thesis, Univ. Vigo.

Reina-Hervás J.A., J.L. Rueda, J.E. García Muñoz and J.E. García Raso. - 2006. Estructura de una población íctica asociada a una pradera de Zostera marina en el Mar de Alborán (Mediterráneo Occidental). In: M. Ballesteros, C. Palacin and X. Turon (eds.), XIV Simposio Ibérico de Estudios de Biología Marina, p. 195. Universitat de Barcelona, Barcelona.

Rolán, E.M. - 1983. Moluscos de la ría de Vigo 1. Gasterópodos. Thalassas, Anexo 1: 1-383.

Rolán, E.M., J. Otero Schmitt and E. Rolán Álvarez. - 1990. Moluscos de la ría de Vigo 2. Poliplacóforos, Bivalvos, Escafópodos, Cefalópodos. Thalassas, Anexo 2: 1-276.

Rueda, J.L., C. Salas and S. Gofas. - 2000. A molluscan community from bioclastic bottoms in the Strait of Gibraltar area. Iberus, 18: $95-123$.

Rueda, J.L., M. Fernández-Casado, C. Salas and S. Gofas. - 2001. Seasonality in a taxocenosis of molluscs from soft bottoms in the Bay of Cádiz (southern Spain). J. Mar. Biol. Assoc. U.K., 81: 903-912.

Rueda, J.L. and C. Salas. - 2003a. Seasonal variation of a molluscan assemblage living in a Caulerpa prolifera meadow within the inner bay of Cádiz (SW Spain). Estuar. Coast. Shelf Sci., 57: 909-918.

Rueda, J.L. and C. Salas. - 2003b. Temporal dynamics of molluscan assemblages from soft and bioclastic bottoms in the Strait of Gibraltar. Cah. Biol. Mar., 44: 237-248.

Rueda, J.L. and C. Salas. - 2007. Trophic dependence of the emerald neritid Smaragdia viridis (Linnaeus, 1758) on two seagrasses from European coasts. J. Moll. Stud., 73: 211-214.

Rueda, J.L. and C. Salas. - 2008. Molluscs associated with a subtidal Zostera marina L. bed in southern Spain: Linking seasonal changes of fauna and environmental variables. Estuar. Coast. Shelf Sci., 79: 157-167.

Rueda, J.L. - 2007. Malacofauna asociada a praderas de Zostera marina del Paraje Natural "Acantilados de Maro-Cerro Gordo" (Sur España). Ph.D. thesis, Univ. Málaga.

Rueda, J.L., C. Salas and P. Marina. - 2008a. Seasonal dynamics of a deep subtidal Zostera marina L. bed in southern Spain (western Mediterranean Sea). Bot. Mar. 51: 92-102.

Rueda, J.L., J. Urra and C. Salas. - 2008b. Diel and seasonal variation of a molluscan taxocoenosis associated with a Zostera marina bed in southern Spain (Alboran Sea). Helgol. Mar. Res. 62: $227-240$.

Russell-Hunter, W.D. - 1983. The Mollusca. Vol. 6. Ecology. Academic Press, Orlando.

Russo, G.F., E. Fresi, D. Vinci and L.A. Chessa. - 1984. Mollusk syntaxon of foliar stratum along a depth gradient in a Posidonia oceanica (L.) Delile meadow: Seasonal variability. In: C.F. Boudouresque, A. Jeudy de Grissac and J. Olivier (eds), International Workshop Posidonia oceanica Beds, pp. 311-318. GIS Posidonie Publ., Marseille.

Scipione, M.B., M.C. Gambi, M. Lorenti, G.F. Russo and V. Zupo. - 1996. Vagile fauna of the leaf stratum of Posidonia oceanica and Cymodocea nodosa in the Mediterranean Sea. In: J. Kuo, R.C. Phillips, D.I. Walker and H. Kirkman (eds.), Seagrass biology: Proceedings of an International Workshop, pp. 249-260. Rottnest Island, Western Australia.

Sfriso, A., T. Birkemeyer and P.F. Ghetti. - 2001. Benthic macrofauna changes in areas of Venice lagoon populated by seagrasses or seaweeds. Mar. Environ. Res., 52: 323-349.

Tebble, N. - 1966. British Bivalve Seashells: A handbook for identification. Royal Scottish Museum, Trustees of the British Museum (Natural History), Edinburgh.

Templado, J. - 1982. Moluscos de las formaciones de fanerógamas marinas en las costas del Cabo de Palos (Murcia). Ph.D. thesis, Univ. Complutense de Madrid.

Templado, J. - 1984a. Moluscos de las praderas de Posidonia oceanica en las costas del Cabo de Palos (Murcia). Invest. Pesq., 48: 509-526. 
Templado, J. - 1984b. Las praderas de Posidonia oceanica en el sureste Español y su biocenosis. In: C.F. Boudouresque, A. Jeudy de Grissac and J. Olivier (eds.), International workshop Posidonia oceanica beds, pp. 159-172. GIS Posidonie Publ., Marseille.

Templado, J., A. Guerra, J. Bedoya, D. Moreno, J.M. Remón, M. Maldonado and M.A. Ramos. - 1993. Fauna marina circalitoral del Sur de la Península Ibérica: Resultados de la campaña oceanográfica "Fauna 1". Museo Nacional de Ciencias Naturales. CSIC, Madrid.

Terlizzi, A. and G.F. Russo. - 1995. Variabilità strutturale del popolamento a molluschi di alcune praterie di Posidonia Oceanica del Mediterraneo. Biol. Mar. Med., 2: 423-426.

Terlizzi, A. and G.F. Russo. - 1997. The molluscan taxocoenose of differently-exposed Cymodocea nodosa beds: year-long structural patterns and sampling methods. Boll. Malacol., 33: 77-82.

Thayer, G.W., S.M. Adams and M.W. LaCroix. - 1975. Structural and functional aspects of a recently established Zostera marina community. In: L.E. Cronin (ed.), Estuarine Research, vol. 1, pp. 517-540. Academic Press, New York.

Thompson, T.E. and G.H. Brown. - 1976. British opisthobranch molluscs. Keys and notes for the identification of species. Academic Press, London.

Toyohara, T., M. Nakaoka and K. Aioi. - 1999. Population dynamics and reproductive traits of phytal gastropods in seagrass bed in Otsuchi Bay, north-eastern Japan. P.S.Z.N.I. Mar. Ecol., 20: 273-289.

Urra, J., P. Marina and J.L. Rueda. - 2008. S.O.S por las praderas de fanerógamas marinas en la Costa del Sol. Quercus, 270: 28-37.

Scient. ed.: M. Gaspar.

Received September 2, 2008. Accepted January 28, 2009.

Published online July 6, 2009. 